\title{
Grothendieck's Theorem \\ for Operator Spaces
}

\author{
by Gilles Pisier* \\ Texas A\&M University and Université Paris 6 \\ and \\ Dimitri Shlyakhtenko** \\ University of California at Los Angeles
}

\begin{abstract}
We prove several versions of Grothendieck's Theorem for completely bounded linear maps $T: E \rightarrow F^{*}$, when $E$ and $F$ are operator spaces. We prove that if $E, F$ are $C^{*}$ algebras, of which at least one is exact, then every completely bounded $T: E \rightarrow F^{*}$ can be factorized through the direct sum of the row and column Hilbert operator spaces. Equivalently $T$ can be decomposed as $T=T_{r}+T_{c}$ where $T_{r}$ (resp. $T_{c}$ ) factors completely boundedly through a row (resp. column) Hilbert operator space. This settles positively (at least partially) some earlier conjectures of Effros-Ruan and Blecher on the factorization of completely bounded bilinear forms on $C^{*}$-algebras. Moreover, our result holds more generally for any pair $E, F$ of "exact" operator spaces. This yields a characterization of the completely bounded maps from a $C^{*}$-algebra (or from an exact operator space) to the operator Hilbert space $\mathrm{OH}$. As a corollary we prove that, up to a complete isomorphism, the row and column Hilbert operator spaces and their direct sums are the only operator spaces $E$ such that both $E$ and its dual $E^{*}$ are exact. We also characterize the Schur multipliers which are completely bounded from the space of compact operators to the trace class.
\end{abstract}

\footnotetext{
* Partially supported by NSF and Texas Advanced Research Program 010366-163

** Partially supported by NSF and Sloan fellowship
} 
Introduction. In 1956, Grothendieck published the fascinating paper [G] now often referred to as "the Résumé". The central result there was described by Grothendieck as "the fundamental theorem of the metric theory of tensor products". This result, now known as Grothendieck's theorem (GT in short) -or sometimes "Grothendieck's inequality" - has played a major role in the developments of Banach space theory in the last three decades; moreover, its non-commutative version has also found important applications to several specific questions in $C^{*}$-algebra theory (see [P5]). It is natural to wonder whether this result still holds, at least in some form, for the recently introduced and currently very active noncommutative analogue of Banach spaces, namely "operator spaces", in the sense of [BP, ER2] (see also [ER1, P1]), and this is precisely the goal of the present paper. Let $A, B$ be $C^{*}$-algebras. While the previous versions are all concerned with bounded bilinear forms on $A \times B$, or equivalently bounded linear maps $T: A \rightarrow B^{*}$, and their possible bounded factorizations, we will study completely bounded bilinear forms or equivalently completely bounded linear maps $T: A \rightarrow B^{*}$, and their possible completely bounded factorizations. For instance, the classical GT and its later extensions say that any bounded $T: A \rightarrow B^{*}$ factors boundedly through a Hilbert space. One of our main results says that, under a mild restriction on either $A$ or $T$, any completely bounded $T: A \rightarrow B^{*}$ factors completely boundedly through the direct sum of two very simple "building blocks": the row and column Hilbert operator spaces. We can thus claim that while GT entirely elucidates the bounded case, we analogously elucidate the completely bounded one.

We will now describe more precisely the connections of our work with the existing literature and the conjectures which motivated it.

The non-commutative version of Grothendieck's theorem says that, if $A, B$ are $C^{*}$-algebras any bounded bilinear form $u: A \times B \rightarrow \mathbb{C}$ satisfies the following inequality: for any finite sequence $\left(a_{i}, b_{i}\right)$ in $A \times B$ we have

$$
\left|\sum u\left(a_{i}, b_{i}\right)\right| \leq K\|u\|\left\{\left\|\sum a_{i}^{*} a_{i}\right\|+\left\|\sum a_{i} a_{i}^{*}\right\|\right\}^{1 / 2}\left\{\left\|\sum b_{i}^{*} b_{i}\right\|+\left\|\sum b_{i} b_{i}^{*}\right\|\right\}^{1 / 2}
$$

where $K$ is a numerical constant (independent of $A, B$ and $u$ ).

Moreover, by a rather simple application of the Hahn-Banach theorem, (0.1) implies that 
there are states $f_{1}, f_{2}$ on $A$ and $g_{1}, g_{2}$ on $B$ such that

$$
\forall(a, b) \in A \times B \quad|u(a, b)| \leq K\|u\|\left\{f_{1}\left(a a^{*}\right)+f_{2}\left(a^{*} a\right)\right\}^{1 / 2}\left\{g_{2}\left(b b^{*}\right)+g_{1}\left(b^{*} b\right)\right\}^{1 / 2}
$$

This was proved for commutative $C^{*}$-algebras by Grothendieck. The non-commutative case was obtained in [P4] with an approximability assumption and in [H1] in full generality. It is easy to deduce from (0.2) that, assuming $\|u\| \leq 1$ for simplicity, there is a decomposition $u=u_{1}+u_{2}+u_{3}+u_{4}$ with

$$
\begin{aligned}
& \left|u_{1}(a, b)\right| \leq K\left\{f_{1}\left(a a^{*}\right)\right\}^{1 / 2}\left\{g_{1}\left(b^{*} b\right)\right\}^{1 / 2} \quad\left|u_{2}(a, b)\right| \leq K\left\{f_{2}\left(a^{*} a\right)\right\}^{1 / 2}\left\{g_{2}\left(b b^{*}\right)\right\}^{1 / 2} \\
& \left|u_{3}(a, b)\right| \leq K\left\{f_{1}\left(a a^{*}\right)\right\}^{1 / 2}\left\{g_{2}\left(b b^{*}\right)\right\}^{1 / 2} \quad\left|u_{4}(a, b)\right| \leq K\left\{f_{2}\left(a^{*} a\right)\right\}^{1 / 2}\left\{g_{1}\left(b^{*} b\right)\right\}^{1 / 2}
\end{aligned}
$$

In view of the recent development of operator space theory, it is natural to look for a version of this theorem for jointly completely bounded (j.c.b. in short) bilinear forms. But here the terminology poses a problem, there are two different notions in the bilinear case: the joint complete boundedness ([ER1-2,BP]) and the complete boundedness in Christensen and Sinclair's sense ([CS1-2, CES]). The second notion came first, was then called simply "complete boundedness" and has proved extremely fruitful. To (reluctantly) conform with the already established tradition, we will call the first ones "jointly c.b." although calling them c.b. would definitely be more natural from the viewpoint of operator space theory. Indeed, given a $C^{*}$-algebra or more generally an operator space $F \subset B(H)$, the latter theory provides us with a natural realization of the dual $F^{*}$ as an operator space, so that we have a specific isometric embedding $F^{*} \subset B(\mathcal{H})$. Thus, given two $C^{*}$-algebras $A, B$ and operator spaces $E \subset A$ and $F \subset B$, we say that a bilinear form $u: E \times F \rightarrow \mathbb{C}$ is jointly completely bounded (in short j.c.b.) if the associated linear map $\widetilde{u}: E \rightarrow F^{*} \subset B(\mathcal{H})$ is c.b. Moreover we let

$$
\|u\|_{j c b}=\|\widetilde{u}\|_{c b\left(E, F^{*}\right)} .
$$

Using tensor products, this definition can be extended to the case of bilinear forms $u$ with values in $B(\mathcal{H})$, the preceding case then corresponds to $\operatorname{dim}(\mathcal{H})=1$. See $\S 1$ for details. 
Now a bilinear map $u: E \times F \rightarrow \mathbb{C}$ is called c.b. (in Christensen and Sinclair's sense) if the bilinear forms

$$
\begin{gathered}
u_{n}: M_{n}(E) \times M_{n}(F) \rightarrow M_{n} \\
\left(\left(a_{i j}\right),\left(b_{i j}\right)\right) \rightarrow\left(\sum_{k} u\left(a_{i k}, b_{k j}\right)\right)_{i j}
\end{gathered}
$$

are uniformly bounded, and by definition

$$
\|u\|_{c b}=\sup _{n \geq 1}\left\|u_{n}\right\|
$$

Here again, this definition can be extended to the case of bilinear forms $u$ with values in $B(\mathcal{H})$. See $\S 1$ for more on this.

This notion is much better understood than the preceding one. In particular, it is easy to show that a bilinear form $u: E \times F \rightarrow \mathbb{C}$ is c.b. with $\|u\|_{c b} \leq 1$ iff for all finite sequence $\left(a_{i}, b_{i}\right)$ in $E \times F$ we have

$$
\left|\sum u\left(a_{i}, b_{i}\right)\right| \leq\left\|\sum a_{i} a_{i}^{*}\right\|^{1 / 2}\left\|\sum b_{i}^{*} b_{i}\right\|^{1 / 2} .
$$

Moreover, assuming $E \subset A$ and $F \subset B$, then (0.5) implies that there are states $f_{1}, g_{1}$ on $A$ and $B$ respectively such that

$$
\forall(a, b) \in E \times F \quad|u(a, b)| \leq\left(f_{1}\left(a a^{*}\right) g_{1}\left(b^{*} b\right)\right)^{1 / 2} .
$$

(Note that conversely $(0.5)^{\prime} \Rightarrow(0.5)$ so $(0.5)$ and $(0.5)^{\prime}$ are essentially equivalent.) Moreover there is an extension of $u$ to $A \times B$ with the same c.b. norm.

When $u: E \times F \rightarrow \mathbb{C}$ is c.b. the associated linear map $\widetilde{u}: E \rightarrow F^{*}$ admits a factorization of the form $E \stackrel{v}{\longrightarrow} H_{r} \stackrel{w}{\longrightarrow} F^{*}$ through a row Hilbert space with $\widetilde{u}=w v$ and $\|v\|_{c b}\|w\|_{c b}=$ $\|u\|_{c b}([\mathrm{ER} 3])$. In particular, we have

$$
\|\widetilde{u}\|_{c b} \leq\|u\|_{c b}
$$

or equivalently

$$
\|u\|_{j c b} \leq\|u\|_{c b} .
$$


It is worthwhile to observe that the Christensen-Sinclair notion is not symmetric:

if $u: E \times F \rightarrow \mathbb{C}$ is c.b. the transposed bilinear form ${ }^{t} u: F \times E \rightarrow \mathbb{C}$ is not necessarily c.b., while for j.c.b. forms this is true and we do have

$$
\left\|{ }^{t} u\right\|_{j c b}=\|u\|_{j c b}
$$

(This is related to the basic fact from operator space theory that $\|\widetilde{u}\|_{c b}=\left\|\widetilde{u}^{*}\right\|_{c b}$.) Thus (0.6) implies for any bilinear from $v: E \times F \rightarrow \mathbb{C}$

$$
\|v\|_{j c b} \leq\left\|{ }^{t} v\right\|_{c b}
$$

and more generally (0.6) and (0.7) together yield

$$
\|u+v\|_{j c b} \leq\|u\|_{c b}+\left\|{ }^{t} v\right\|_{c b}
$$

We note that (see (0.5) above) $\left\|{ }^{t} v\right\|_{c b} \leq 1$ iff for any finite sequence $\left(a_{i}, b_{i}\right)$ in $E \times F$ we have

$$
\left|\sum v\left(a_{i}, b_{i}\right)\right| \leq\left\|\sum a_{i}^{*} a_{i}\right\|^{1 / 2}\left\|\sum b_{i} b_{i}^{*}\right\|^{1 / 2}
$$

or equivalently iff there are states $f_{2}, g_{2}$ on $A$ and $B$ respectively such that

$$
\left.\forall(a, b) \in E \times F \quad|v(a, b)| \leq\left(f_{2}\left(a^{*} a\right) g_{2}\left(b b^{*}\right)\right)\right)^{1 / 2} .
$$

Thus if $U=u+v$ with $\|u\|_{c b} \leq 1$ and $\left\|{ }^{t} v\right\|_{c b} \leq 1$ we find states $f_{1}, g_{1}, f_{2}, g_{2}$ such that

$$
\forall(a, b) \in E \times F \quad|U(a, b)| \leq\left(f_{1}\left(a a^{*}\right) g_{1}\left(b^{*} b\right)\right)^{1 / 2}+\left(f_{2}\left(a^{*} a\right) g_{2}\left(b b^{*}\right)\right)^{1 / 2}
$$

or equivalently for all finite sequences $\left(a_{i}, b_{i}\right) \in E \times F$ and all $\lambda_{i}>0$

$$
(0.10)^{\prime}\left|\sum U\left(a_{i}, b_{i}\right)\right| \leq\left\|\sum a_{i} a_{i}^{*}\right\|^{1 / 2}\left\|\sum b_{i}^{*} b_{i}\right\|^{1 / 2}+\left\|\sum \lambda_{i} a_{i}^{*} a_{i}\right\|^{1 / 2}\left\|\sum \lambda_{i}^{-1} b_{i} b_{i}^{*}\right\|^{1 / 2} .
$$

Conversely it can be shown that (0.10) implies the existence of decomposition $U=u+v$ with $\max \left\{\|u\|_{c b},\left\|{ }^{t} v\right\|_{c b}\right\} \leq 1$. (The proof of this converse is less obvious than may seem at first glance. We give the details in $\S 2$ below. We show there that $(0.10)$ implies a decomposition $U=u+v$ together with states $f_{1}^{\prime}, f_{2}^{\prime}, g_{1}^{\prime}, g_{2}^{\prime}$ such that

$$
|u(a, b)| \leq\left(f_{1}^{\prime}\left(a a^{*}\right) g_{1}^{\prime}\left(b^{*} b\right)\right)^{1 / 2} \text { and }|v(a, b)| \leq\left(f_{2}^{\prime}\left(a^{*} a\right) g_{2}^{\prime}\left(b b^{*}\right)\right)^{1 / 2}
$$


but we apparently cannot do this with the original states $f_{1}, f_{2}, g_{1}, g_{2}$.)

We now return to the above decomposition $u=u_{1}+u_{2}+u_{3}+u_{4}$. There $u_{1}$ and $u_{2}$ are jointly c.b., but $u_{3}$ and $u_{4}$ in general are not. More precisely, $u_{1}+u_{2}$ is jointly c.b. on $A \times B$, while $u_{3}+u_{4}$ is jointly c.b. on $A \times B^{o p}$, where $B^{o p}$ denotes the opposite $C^{*}$-algebra (anti-isomorphic to $B$ ). Probably led by similar observations, Effros and Ruan formulated in [ER2] (with $K=1$ ) the following

Conjecture 0.1. Let $A, B$ be $C^{*}$-algebras and let $u: A \times B \rightarrow \mathbb{C}$ be a j.c.b. bilinear form. Then there exist states $f_{1}, f_{2}, g_{1}, g_{2}$ on $A, B$ respectively such that

$\forall(a, b) \in A \times B \quad|u(a, b)| \leq K\|u\|_{j c b}\left(f_{1}\left(a a^{*}\right) g_{1}\left(b^{*} b\right)\right)^{1 / 2}+\left(f_{2}\left(a^{*} a\right) g_{2}\left(b b^{*}\right)\right)^{1 / 2}$

where $K$ is a numerical constant.

Independently, Blecher [B1] was led to a similar conjecture:

Conjecture 0.2. Let $A=B(\mathcal{H})$ and $B=B(\mathcal{K})$ where $\mathcal{H}, \mathcal{K}$ are Hilbert spaces. There is a constant $K$ such that for any $w$ in the algebraic tensor product $A \otimes B$ we have

$$
\|w\|_{\wedge} \leq K \max \left\{\|w\|_{h},\left\|^{t} w\right\|_{h}\right\}
$$

where $\|w\|_{\wedge}$ is the operator space version of the projective norm and where $\|w\|_{h}$ (resp. $\left.\left\|{ }^{t} w\right\|_{h}\right)$ is the norm in the Haagerup tensor product $A \otimes_{h} B$ (resp. $\left.B \otimes_{h} A\right)$.

In the unpublished problem book of a 1993 conference, he also asked the same questions when $A, B$ are $C^{*}$-algebras. Moreover although he did not make it precise, Blecher implicitly conjectured that the corresponding matricial norms were uniformly equivalent, so that the associated tensor products $A \otimes^{\wedge} B$ and $\left[A \otimes_{h} B\right] \cap{ }^{t}\left[B \otimes_{h} A\right]$ should be completely isomorphic. At the time of this writing, we are unable to prove this (even when $A$ and $B$ are both commutative!).

By [BP, ER2] we know that if $A, B$ are arbitrary operator spaces, we have

$$
\|w\|_{\wedge}=\sup \{|\langle w, U\rangle|\}
$$


where the supremum runs over all bilinear forms $U: A \times B \rightarrow \mathbb{C}$ with $\|U\|_{j c b} \leq 1$, and also

$$
\|w\|_{A \otimes_{h} B}=\sup \{|\langle w, u\rangle|\}
$$

where the supremum runs over all bilinear forms $u: A \times B \rightarrow \mathbb{C}$ with $\|u\|_{c b} \leq 1$. Thus Conjecture 0.2 is equivalent by duality to the following

Conjecture 0.2'. There is a constant $K$ such that any j.c.b. bilinear form $U: A \times B \rightarrow \mathbb{C}$ can be decomposed as a sum $U=u+v$ with $\|u\|_{c b}+\left\|{ }^{t} v\right\|_{c b} \leq K\|U\|_{j c b}$.

In other words Blecher conjectured that the estimate (0.8) can be reversed on $A \times B$, thus establishing a very nice (and simple) relationship between the two notions of c.b. for bilinear forms.

Our main objective in this paper is to prove versions of these conjectures. More precisely, we will prove Conjectures 0.1 and 0.2 ' assuming either $A$ or $B$ is exact, or for arbitrary $C^{*}$-algebras but under a suitable approximability assumption on the bilinear form $u$. Actually, our results are valid for j.c.b. bilinear forms $U: E \times F \rightarrow \mathbb{C}$ defined on exact operator spaces. In particular, we will prove that, assuming $E \subset A$ and $F \subset B$, any such map extends to a j.c.b. bilinear form $\widehat{U}$ defined on $A \times B$. Unfortunately, since $B(H)$ is not exact, we cannot prove Conjecture 0.2 as stated above. Also, the extension of Conjecture 0.2 ' to the case of a $B(H)$-valued bilinear form remains open at the time of this writing.

Our results constitute a sequel to the papers [JP] and [P3].

We say that an operator space $E$ is exact if there is a constant $C$ such that for any finite dimensional subspace $G \subset E$ there is an integer $N$, a subspace $\widetilde{G} \subset M_{N}$ and an isomorphism $u: G \rightarrow \widetilde{G}$ such that $\|u\|_{c b}\left\|u^{-1}\right\|_{c b} \leq C$. We will denote by $e x(E)$ the smallest $C$ for which this holds. This is the operator space analog of a notion introduced and extensively studied by Kirchberg for $C^{*}$-algebras $([\mathrm{Ki} 3])$.

In $[\mathrm{JP}]$, a characterization and an extension theorem were obtained for "tracially" bounded bilinear forms $u: E \times F \rightarrow \mathbb{C}$ when $E, F$ are exact operator spaces. "Tracial boundedness" is a notion intermediate between boundedness and complete boundedness.

The precise statements of our main results are as follows. 
Theorem 0.3. Let $E \subset A, F \subset B$ be exact operator spaces sitting in $C^{*}$-algebras $A, B$ with exactness constants respectively ex $(E)$ and ex $(F)$. Let $C=e x(E) e x(F)$. Then any j.c.b. bilinear form $U: E \times F \rightarrow \mathbb{C}$ satisfies the following inequality: for any finite sequence $\left(a_{i}, b_{i}\right)$ in $E \times F$ and for any $\lambda_{i}>0$ we have

$$
\begin{gathered}
\left|\sum U\left(a_{i}, b_{i}\right)\right| \leq \\
C\|u\|_{j c b}\left[\left\|\sum \lambda_{i} a_{i}^{*} a_{i}\right\|^{1 / 2}+\left\|\sum \lambda_{i}^{-1} a_{i} a_{i}^{*}\right\|^{1 / 2}\right]\left[\left\|\sum \lambda_{i} b_{i}^{*} b_{i}\right\|^{1 / 2}+\left\|\sum \lambda_{i}^{-1} b_{i} b_{i}^{*}\right\|^{1 / 2}\right]
\end{gathered}
$$

and consequently:

$$
\left|\sum U\left(a_{i}, b_{i}\right)\right| \leq 2 C\|u\|_{j c b}\left[\left\|\sum a_{i} a_{i}^{*}\right\|^{1 / 2}\left\|\sum b_{i}^{*} b_{i}\right\|^{1 / 2}+\left\|\sum a_{i}^{*} a_{i}\right\|^{1 / 2}\left\|\sum b_{i} b_{i}^{*}\right\|^{1 / 2}\right] .
$$

Theorem 0.4. Let $K=2^{3 / 2} C$. Then for any j.c.b. bilinear form $U: E \times F \rightarrow \mathbb{C}$ with $\|U\|_{j c b} \leq 1$, there are states $f_{1}, f_{2}$ and $g_{1}, g_{2}$ on $A, B$ respectively such that

$$
\forall(a, b) \in E \times F \quad|U(a, b)| \leq K\left[\left(f_{1}\left(a a^{*}\right) g_{1}\left(b^{*} b\right)\right)^{1 / 2}+\left(f_{2}\left(a^{*} a\right) g_{2}\left(b b^{*}\right)\right)^{1 / 2}\right] .
$$

Moreover, for any finite sequence $\left(a_{i}, b_{i}\right)$ in $E \times F$ and for any $\lambda_{i}>0$ we have

$$
\left|\sum U\left(a_{i}, b_{i}\right)\right| \leq K\left[\left\|\sum a_{i} a_{i}^{*}\right\|^{1 / 2}\left\|\sum b_{i}^{*} b_{i}\right\|^{1 / 2}+\left\|\sum \lambda_{i} a_{i}^{*} a_{i}\right\|^{1 / 2}\left\|\sum \lambda_{i}^{-1} b_{i} b_{i}^{*}\right\|^{1 / 2}\right] .
$$

Finally, any form $U$ satisfying (0.15) for some $K$ can be decomposed as $U=u+v$ where $u, v$ are bilinear forms satisfying

$$
\max \left\{\|u\|_{h},\left\|{ }^{t} v\right\|_{h}\right\} \leq K .
$$

Theorem 0.5. Let $A, B$ be $C^{*}$-algebras. Let $U: A \times B \rightarrow \mathbb{C}$ be a bilinear form with $\|U\|_{j c b} \leq 1$. Either one of the two following assumptions ensures that $U$ satisfies the conclusions of Theorem 0.4 with $K=2^{3 / 2}$.

(i) At least one of the algebras $A, B$ is exact.

(ii) The form $U$ is the pointwise limit of a net of finite rank forms $U_{\alpha}$ such that $\left\|U_{\alpha}\right\|_{j c b} \leq 1$.

Corollary 0.6. In the same situation as Theorem 0.4, any j.c.b. bilinear form $U: E \times F \rightarrow$ $\mathbb{C}$ admits an extension $\widehat{U}: A \times B \rightarrow \mathbb{C}$ with $\|\widehat{U}\|_{j c b} \leq K\|U\|_{j c b}$.

Our result admits a nice reformulation as a factorization involving the row and column operator space structures (resp. $H_{r}$ and $H_{c}$ ) on a Hilbert space $H$ : 
Corollary 0.7. In the situation of Theorem 0.4, any c.b. linear map $T: E \rightarrow F^{*}$ can be factorized completely boundedly through an operator space of the form $H_{r} \oplus H_{c}$ for some Hilbert space $H$. More precisely there are c.b. maps $v: E \rightarrow H_{r} \oplus H_{c}$ and $w: H_{r} \oplus H_{c} \rightarrow F^{*}$ such that $T=w v$ and $\|w\|_{c b}\|v\|_{c b} \leq 2^{3 / 2} C\|T\|_{c b}$. On the other hand, in the situation of Theorem 0.5, any c.b. linear map $T: A \rightarrow B^{*}$ which can be approximated by finite rank completely contractive maps, in the point weak-* topology, can be factorized in the same way, $T=w v$ with $\|w\|_{c b}\|v\|_{c b} \leq 2^{3 / 2}$; moreover, the approximability assumption on $T$ can be dispensed with if either $A$ or $B$ is exact.

In the "predual" situation, our results also yield:

Corollary 0.8. Let $E, F$ be exact operator spaces and let $C=\operatorname{ex}(E) \operatorname{ex}(F)$ as before. Let $A, B$ be arbitrary $C^{*}$-algebras. Then the following isomorphisms hold:

(i) $E \otimes^{\wedge} F \simeq\left[E \otimes_{h} F\right] \cap\left[{ }^{t}\left(F \otimes_{h} E\right)\right]$. Here $E \otimes^{\wedge} F$ denotes the operator space version of the projective tensor product.

(ii) $A \otimes^{\wedge} B \simeq\left[A \otimes_{h} B\right] \cap\left[{ }^{t}\left(B \otimes_{h} A\right)\right]$.

(iii) $A^{*} \otimes_{\min } B^{*} \simeq A^{*} \otimes_{h} B^{*}+{ }^{t}\left(B^{*} \otimes_{h} A^{*}\right)$.

The equivalence constant is $\leq 4 \sqrt{2} C$ in (i) and $\leq 4 \sqrt{2}$ in (ii) and (iii).

\section{$\S 1$. Background}

We refer to [Pa1] (resp. [CS1-2]) for background on c.b. linear (resp. multilinear) maps and to [ER1] and [P1] for background on operator spaces in general. We just recall that the minimal tensor product $E \otimes_{\min } F$ of two operator spaces $E \subset B\left(H_{1}\right), F \subset B\left(H_{2}\right)$ is defined so that we have a completely isometric embedding

$$
E \otimes_{\min } F \subset B\left(H_{1} \otimes_{2} H_{2}\right)
$$

We now give a precise definition for "joint" complete boundedness.

Definition 1.1. Let $E, F, G$ be operator spaces. A bilinear form $u$ : $E \times F \rightarrow G$ is j.c.b. if for any $C^{*}$-algebras $B_{1}, B_{2} u$ can be "extended" to a bounded bilinear form $(u)_{B_{1}, B_{2}}: E \otimes_{\min } B_{1} \times F \otimes_{\min } B_{2} \rightarrow G \otimes_{\min } B_{1} \otimes_{\min } B_{2}$ taking $\left(e \otimes b_{1}, f \otimes b_{2}\right)$ to $u(e, f) \otimes b_{1} \otimes b_{2}$. Moreover we have

$$
\|u\|_{j c b}=\sup \left\|(u)_{B_{1}, B_{2}}\right\|
$$


where the supremum runs over all pairs $B_{1}, B_{2}$ of $C^{*}$-algebras.

Equivalently, we have $\|u\|_{j c b} \leq 1$ iff for all $C^{*}$-algebras $B_{1}, B_{2}$ and for all finite sums

$$
\sum a_{i} \otimes x_{i} \in E \otimes B_{1}, \quad \sum b_{j} \otimes y_{j} \in F \otimes B_{2}
$$

we have

$$
\left\|\sum u\left(a_{i}, b_{j}\right) \otimes x_{i} \otimes y_{j}\right\|_{\min } \leq\left\|\sum a_{i} \otimes x_{i}\right\|_{\min }\left\|\sum b_{j} \otimes y_{j}\right\|_{\min } .
$$

In addition, it suffices to consider matricial $C^{*}$-algebras for $B_{1}$ and $B_{2}$, more precisely we have actually

$$
\|u\|_{j c b}=\sup _{n \geq 1}\left\|(u)_{M_{n}, M_{n}}\right\|
$$

It is easy to see that the usual definition of $\|u\|_{j c b}$ (which is $\|u\|_{j c b}=\|\widetilde{u}\|_{c b\left(E, F^{*}\right)}$ ) is equivalent to (1.2). The equality of (1.1) and (1.2) is a routine verification left to the reader.

Following the same pattern we have the following equivalent definition for complete boundedness:

Proposition 1.2. A bilinear form $u: E \times F \rightarrow G$ is c.b. iff for any $C^{*}$-algebra $A$, $u$ can be "extended" to a bounded bilinear form $u_{A}: E \otimes_{\min } A \times F \otimes_{\min } A \rightarrow G \otimes_{\min } A$ taking $\left(e \otimes a_{1}, f \otimes a_{2}\right)$ to $u(e, f) \otimes a_{1} a_{2}$. Moreover we have

$$
\|u\|_{c b}=\sup \left\|u_{A}\right\|
$$

where the supremum runs over all possible $C^{*}$-algebras $A$ and actually

$$
\|u\|_{c b}=\sup _{n \geq 1}\left\|u_{M_{n}}\right\|
$$

The equivalences $(0.1) \Leftrightarrow(0.1)^{\prime},(0.5) \Leftrightarrow(0.5)^{\prime},(0.9) \Leftrightarrow \quad(0.9)^{\prime}$ all follow from the same Hahn-Banach type argument as follows. 
Proposition 1.3. Let $A, B$ be $C^{*}$-algebras. Assume $E \subset A, F \subset B$. Let $u: E \times F \rightarrow \mathbb{C}$ be a bilinear form. Let $\alpha_{1}, \alpha_{2}$ and $\theta_{1}, \theta_{2}$ be fixed non-negative numbers. Then the following assertions are equivalent

(i) There are states $\left(f_{1}, f_{2}\right),\left(g_{1}, g_{2}\right)$ on $A$ and $B$ respectively such that for all $(a, b)$ in $E \times F$ we have

$$
|u(a, b)| \leq\left[\alpha_{1} f_{1}\left(a^{*} a\right)+\alpha_{2} f_{2}\left(a a^{*}\right)\right]^{1 / 2}\left[\theta_{2} g_{2}\left(b^{*} b\right)+\theta_{1} g_{1}\left(b b^{*}\right)\right]^{1 / 2} .
$$

(ii) For all finite sequences $\left(a_{i}, b_{i}\right)$ in $E \times F$ we have

$$
\left|\sum u\left(a_{i}, b_{i}\right)\right| \leq\left[\alpha_{1}\left\|\sum a_{i}^{*} a_{i}\right\|+\alpha_{2}\left\|\sum a_{i} a_{i}^{*}\right\|\right]^{1 / 2}\left[\theta_{2}\left\|\sum b_{i}^{*} b_{i}\right\|+\theta_{1}\left\|\sum b_{i} b_{i}^{*}\right\|\right]^{1 / 2} .
$$

Proof. (sketch) (i) $\Rightarrow$ (ii) is obvious by Cauchy-Schwarz. Conversely assume (ii). We will use the classical arithmetic/geometric mean inequality as follows:

$$
\forall x, y \geq 0 \quad(x y)^{1 / 2} \leq 2^{-1}(x+y) \text { and }(x y)^{1 / 2}=\inf _{\lambda>0} 2^{-1}\left(\lambda x+\lambda^{-1} y\right) .
$$

Thus (ii) and (1.3) imply:

$2\left|\sum u\left(a_{i}, b_{i}\right)\right| \leq \sup \left[\alpha_{1} \sum f_{1}\left(a_{i}^{*} a_{i}\right)+\alpha_{2} \sum f_{2}\left(a_{i} a_{i}^{*}\right)+\theta_{2} \sum g_{2}\left(b_{i}^{*} b_{i}\right)+\theta_{1} \sum g_{1}\left(b_{i} b_{i}^{*}\right)\right]$

where the supremum runs over all pairs of states $\left(f_{1}, f_{2}\right)$ and $\left(g_{1}, g_{2}\right)$ on $A$ and $B$ respectively.

Since the right side does not change if we replace $a_{i}$ by $z_{i} a_{i}$ with $\left|z_{i}\right|=1$, the preceding inequality remains valid with $2 \sum\left|u\left(a_{i}, b_{i}\right)\right|$ in place of $2\left|\sum u\left(a_{i} b_{i}\right)\right|$ on the left side. Then by a well known variant of the "minimax principle", we find states $f_{1}, f_{2}$ and $g_{1}, g_{2}$ such that for all $(a, b)$ in $E \times F$ we have

$$
2|u(a, b)| \leq \alpha_{1} f_{1}\left(a^{*} a\right)+\alpha_{2} f_{2}\left(a a^{*}\right)+\theta_{2} g_{2}\left(b^{*} b\right)+\theta_{1} g_{1}\left(b b^{*}\right)
$$

and finally involving (1.3) again we obtain (i).

1.4. For the reader's convenience, we now summarize the basic facts on the Haagerup tensor product $E \otimes_{h} F$ of two operator spaces $E, F$. Let us denote by $E \otimes F$ the algebraic 
tensor product. Assume $E, F$ are given together with (completely isometric) embeddings $E \subset A$ and $F \subset B$ in $C^{*}$-algebras $A$ and $B$. For any $x=\sum a_{i} \otimes b_{i}$ in $E \otimes F$, we define

$$
\|x\|_{h}=\inf \left\{\left\|\sum a_{i} a_{i}^{*}\right\|^{1 / 2}\left\|\sum b_{i}^{*} b_{i}\right\|^{1 / 2}\right\}
$$

where the infimum runs over all possible ways to write $x$ as a finite sum of the form $x=\sum a_{i} \otimes b_{i}$. We will denote by $E \otimes_{h} F$ the completion of the resulting normed space. More generally, let $x=\left[x_{i j}\right]$ be an $n \times n$ matrix with entries in $E \otimes F$. We define

$$
\|x\|_{(h, n)}=\inf \left\{\|y\|_{M_{n, N}(E)}\|z\|_{M_{N, n}(F)}\right\}
$$

where the infimum runs over all $N \geq 1$ and all possible decompositions of $x$ of the form

$$
x_{i j}=\sum_{k=1}^{N} y_{i k} \otimes z_{k j} .
$$

By known results (see [CS1-2, PaS]), for a suitable $\mathcal{H}$, we can find an isometric embedding of $E \otimes_{h} F$ into $B(\mathcal{H})$ such that the above norm \|\|$_{(h, n)}$ can be identified with the norm induced on $M_{n}(E \otimes F)$ by $M_{n}(B(\mathcal{H}))$. This allows us to think of $E \otimes_{h} F$ as an operator space. Let $K$ be any Hilbert space. Then a bilinear form $u: E \times F \rightarrow B(K)$ is c.b. iff the associated linear map $U: E \otimes_{h} F \rightarrow B(K)$ is well defined and c.b. Moreover we have

$$
\|u\|_{c b}=\|U\|_{c b} .
$$

It is known ([CS1-2, PaS $])$ that $\|u\|_{c b} \leq 1$ iff there is a Hilbert space $H$ and c.b. maps $\sigma_{1}: E \rightarrow B(H, K)$ and $\sigma_{2}: F \rightarrow B(K, H)$ with $\left\|\sigma_{1}\right\|_{c b} \leq 1,\left\|\sigma_{2}\right\|_{c b} \leq 1$ such that $u(a, b)=$ $\sigma_{1}(a) \sigma_{2}(b)$ for any $(a, b)$ in $E \times F$. If $\operatorname{dim}(K)=\infty$, we may actually take $H=K$.

In particular, for a bilinear form $u: E \times F \rightarrow \mathbb{C}$ we find $\|u\|_{c b} \leq 1$ iff there are $H$ together with $\sigma_{1}: E \rightarrow B(H, \mathbb{C})$ and $\sigma_{2}: F \rightarrow B(\mathbb{C}, H)$ such that $u(a, b)=\sigma_{1}(a) \cdot \sigma_{2}(b)$ for all $(a, b)$ in $E \times F$. We will denote by $H_{c}$ and $H_{r}$ the column and row Hilbertian operator spaces. These are defined by

$$
H_{c}=B(\mathbb{C}, H) \quad \text { and } \quad H_{r}=B\left(H^{*}, \mathbb{C}\right)
$$

with the induced operator space structure. Recall (see [ER3]) $\left(H_{c}\right)^{*}=\left(H^{*}\right)_{r}=\bar{H}_{r}$, $\left(H_{r}\right)^{*}=\left(H^{*}\right)_{c}=\bar{H}_{c}$. 
In particular, in the factorization immediately above we have completely contractive linear maps $\sigma_{1}: E \rightarrow \bar{H}_{r}$ and $\sigma_{2}: F \rightarrow H_{c}$. Their adjoints $\sigma_{1}^{*}: H_{c} \rightarrow E^{*}$ and $\sigma_{2}^{*}: \bar{H}_{r} \rightarrow F^{*}$ have the same c.b. norms. Thus, the linear map $\widetilde{u}: E \rightarrow F^{*}$ associated to $u$ can be factorized through $\bar{H}_{r}$, i.e. we can write $\widetilde{u}=\sigma_{2}^{*} \sigma_{1}$. This leads to a description of the space $\left(E \otimes_{h} F\right)^{*}$ in terms of factorization: a bilinear map $u: E \times F \rightarrow \mathbb{C}$ is c.b. iff the linear map $\widetilde{u}: E \rightarrow F^{*}$ admits for some $H$ a c.b. factorization of the form

$$
\widetilde{u}: E \stackrel{\alpha}{\longrightarrow} H_{r} \stackrel{\beta}{\longrightarrow} F^{*} .
$$

Moreover, we have

$$
\|u\|_{c b}=\inf \left\{\|\alpha\|_{c b}\|\beta\|_{c b}\right\}
$$

where the infimum runs over all possible such factorization of $\widetilde{u}$. On the other hand, for the transposed bilinear form ${ }^{t} u: F \times E \rightarrow \mathbb{C}$, we have

$$
\left\|^{t} u\right\|_{c b}=\inf \left\{\|\gamma\|_{c b}\|\delta\|_{c b}\right\}
$$

where the infimum runs over all possible c.b. factorizations of $\widetilde{u}$ of the form

$$
\widetilde{u}: E \stackrel{\gamma}{\longrightarrow} H_{c} \stackrel{\delta}{\longrightarrow} F^{*}
$$

through (this time) a column space $H_{c}$.

1.5. We should mention that $E \otimes_{h} F$ can be realized as a subspace of the full free product $A * B$ of the $C^{*}$-algebras, $A, B$ containing $E, F$ respectively, see [CES]. (This is valid also in the unital case with the unital free product, cf. [P2].) In particular, for any $C^{*}$-algebra $\mathcal{B}$ and any finite sum $x=\sum a_{i} \otimes b_{i} \otimes c_{i}$ in $E \otimes F \otimes \mathcal{B}$ we have

$$
\|x\|_{\left(E \otimes_{h} F\right) \otimes_{\min } \mathcal{B}}=\sup \left\{\left\|\sum \sigma_{1}\left(a_{i}\right) \sigma_{2}\left(b_{i}\right) \otimes c_{i}\right\|_{\min }\right\}
$$

where the supremum runs over all $H$ and all pairs of complete contractions $\sigma_{1}: E \rightarrow B(H)$, $\sigma_{2}: F \rightarrow B(H)$.

1.6. The next result from $[\mathrm{OP}]$ provides a description of the kind of bilinear forms that we encounter in this paper. 
Let $U: E \times F \rightarrow B(K)$ be a bilinear form. The following are equivalent:

(i) There is a decomposition $U=u+v$ with $\|u\|_{c b}+\left\|{ }^{t} v\right\|_{c b} \leq 1$.

(ii) For any $C^{*}$-algebra $\mathcal{B}$ and any finite sum $\sum a_{i} \otimes b_{i} \otimes c_{i}$ in $E \otimes F \otimes \mathcal{B}$ we have

$$
\left\|\sum U\left(a_{i}, b_{i}\right) \otimes c_{i}\right\|_{\min } \leq \sup \left\{\left\|\sum \sigma_{1}\left(a_{i}\right) \sigma_{2}\left(b_{i}\right) \otimes c_{i}\right\|_{\min }\right\}
$$

where the supremum runs over all $H$ and all possible pairs $\sigma_{1}: E \rightarrow B(H), \sigma_{2}: F \rightarrow$ $B(H)$ of complete contractions with commuting ranges.

(iii) $U$ defines a completely contractive linear map from $\left(E \otimes_{h} F\right) \cap\left({ }^{t} F \otimes_{h} E\right)$ equipped with its natural operator space structure.

We will also need the following

Proposition 1.7. Let $E, F$ be operator spaces, and let $w \in E \otimes F$ (algebraic tensor product). Then there is a finite sequence $\left(a_{i}, b_{i}\right),(i=1,2, \ldots, r)$ in $E \times F$ and scalars $\lambda_{i}>0$ such that

$$
\left\|\sum a_{i} a_{i}^{*}\right\|^{1 / 2}\left\|\sum b_{i}^{*} b_{i}\right\|^{1 / 2}=\|w\|_{h}
$$

and

$$
\left\|\sum \lambda_{i} a_{i}^{*} a_{i}\right\|^{1 / 2}\left\|\sum \lambda_{i}^{-1} b_{i}^{*} b_{i}\right\|^{1 / 2}=\left\|^{t} w\right\|_{h} .
$$

Proof. Without loss of generality we may assume $E$ and $F$ finite dimensional. By definition of \|\|$_{h}$ (and a compactness argument) there are $\left(a_{i}, b_{i}\right)$ such that (1.5) holds. Let $\alpha: E^{*} \rightarrow \ell_{2}^{r}$ and $\beta: \ell_{2}^{r} \rightarrow F$ be the linear maps defined by

$$
\alpha(\xi)=\sum \xi\left(a_{i}\right) e_{i} \quad \text { and } \quad \beta\left(e_{i}\right)=b_{i},
$$

so that if $\widetilde{w}: E^{*} \rightarrow F$ denotes the linear map associated to $w$, we have

$$
w=\beta \alpha .
$$

For simplicity we will denote

$$
|\alpha|_{C}=\left\|\sum a_{i}^{*} a_{i}\right\|^{1 / 2} \quad|\alpha|_{R}=\left\|\sum a_{i} a_{i}^{*}\right\|^{1 / 2}
$$


and

$$
|\beta|_{C}=\left\|\sum b_{i}^{*} b_{i}\right\|^{1 / 2} \quad|\beta|_{R}=\left\|\sum b_{i} b_{i}^{*}\right\|^{1 / 2}
$$

We will use repeatedly the following simple observation: for any $\gamma: \ell_{2}^{r} \rightarrow \ell_{2}^{r}$ with $\|\gamma\| \leq 1$, we have

$$
\left\{\begin{array}{l}
|\gamma \alpha|_{R} \leq|\alpha|_{R}, \quad|\gamma \alpha|_{C} \leq|\alpha|_{C} \\
|\beta \gamma|_{R} \leq|\beta|_{R}, \quad|\beta \gamma|_{C} \leq|\beta|_{C}
\end{array}\right.
$$

In particular, this observation allows us to assume that $r$ is the rank of $w$ and that $\left(a_{i}\right)_{i \leq r}$ and $\left(b_{i}\right)_{i \leq r}$ are linearly independent. By the definition of \|\|$_{h}$ again there are maps $\alpha_{1}: E^{*} \rightarrow \ell_{2}^{r}, \beta_{1}: \ell_{2}^{r} \rightarrow F$ such that $\widetilde{w}=\beta_{1} \alpha_{1}$ and $\left|\alpha_{1}\right|_{C}\left|\beta_{1}\right|_{R}=\left\|{ }^{t} w\right\|_{h}$. By the linear independence of $\left(a_{i}\right),\left(b_{i}\right)$, there are linear maps $\gamma$ and $\delta$ on $\ell_{2}^{r}$ such that $\alpha_{1}=\gamma \alpha$ and $\beta_{1}=\beta \delta$. Moreover since $\beta_{1} \alpha_{1}=\beta \alpha$ we must have $\delta \gamma=I$, hence $\delta \equiv \gamma^{-1}$. We now write $\gamma$ as a product

$$
\gamma=\gamma_{1} D \gamma_{2}
$$

where $\gamma_{1}, \gamma_{2}$ are unitary and $D$ is a diagonal matrix with coefficients $D_{i i}>0$.

We have then $\delta=\gamma^{-1}=\gamma_{2}^{-1} D^{-1} \gamma_{1}^{-1}$. Hence we can write

$$
\widetilde{w}=\beta_{1} \alpha_{1}=\left(\beta \gamma_{2}^{-1} D^{-1}\right)\left(D \gamma_{2} \alpha\right)
$$

Now if we replace $\alpha$ and $\beta$ by $\widehat{\alpha}=\gamma_{2} \alpha$ and $\widehat{\beta}=\beta \gamma_{2}^{-1}$ then (1.8) guarantees that (1.5) still holds, but on the other hand, setting $\widehat{\beta}_{1}=\widehat{\beta} D^{-1}$ and $\widehat{\alpha}_{1}=D \widehat{\alpha}$ we now have

$$
\left|\widehat{\alpha}_{1}\right|_{C}=\left|\alpha_{1}\right|_{C} \quad\left|\widehat{\beta}_{1}\right|_{R}=\left|\beta_{1}\right|_{R}
$$

hence $\left|\widehat{\alpha}_{1}\right|_{C}\left|\widehat{\beta}_{1}\right|_{R}=\left\|{ }^{t} w\right\|_{h}$.

Moreover, if we denote by $\left(\widehat{a}_{i}\right)$ and $\left(\widehat{b}_{i}\right)$ the sequences associated to $\widehat{\alpha}$ and $\widehat{\beta}$ as in (1.7) above, then we have

$$
\left\|\sum \widehat{a}_{i} \widehat{a}_{i}^{*}\right\|^{1 / 2}\left\|\sum \widehat{b}_{i}^{*} \widehat{b}_{i}\right\|^{1 / 2}=|\widehat{\alpha}|_{R}|\widehat{\beta}|_{C}=\|w\|_{h}
$$

and on the other hand

$$
\left\|\sum D_{i i}^{2} \widehat{a}_{i}^{*} \widehat{a}_{i}\right\|^{1 / 2}\left\|\sum D_{i i}^{-2} \widehat{b}_{i} \widehat{b}_{i}^{*}\right\|^{1 / 2}=\left|\widehat{\alpha}_{1}\right|_{C}\left|\widehat{\beta}_{1}\right|_{R}=\left\|{ }^{t} w\right\|_{h} .
$$


Hence letting $\lambda_{i}=D_{i i}$ we obtain the announced result.

1.8. A $C^{*}$-algebra $A$ is called WEP (for weak expectation property) if the inclusion map $A \rightarrow A^{* *}$ can be factorized completely contractively through $B(H)$ for some $H$. This notion goes back to Lance [L]. Many equivalent definitions are known. The one we will use is the following characterization due to Kirchberg [Ki2]: let $C$ be the (full) $C^{*}$-algebra of the free group with (say) countably infinitely many generators. Then $A$ is WEP iff $A \otimes_{\min } C=A \otimes_{\max } C$ (that is to say the minimal and maximal $C^{*}$-norms coincide on the algebraic tensor product $A \otimes C)$. A simpler proof was given in [P2].

Following Kirchberg [Ki1], we will say that a $C^{*}$-algebra $A$ is QWEP if it is a quotient of a WEP $C^{*}$-algebra. It is an outstanding open question whether every $C^{*}$-algebra is QWEP. This is equivalent to Connes' question whether every von Neumann algebra (on a separable Hilbert space) embeds into an ultra-product of the hyperfinite factor (see [Ki1] for more on this).

1.9. Let $E$ be a finite dimensional operator space. Let $C=e x(E)$. Then for any $C^{*}$ algebra $B$ and any (closed 2-sided) ideal $I \subset B$, we have a canonical isomorphism

$$
T: E \otimes_{\min }(B / I) \rightarrow\left(E \otimes_{\min } B\right) /\left(E \otimes_{\min } I\right)
$$

with $\|T\| \leq C$ (and obviously $\left\|T^{-1}\right\| \leq 1$ ).

A $C^{*}$-algebra is exact iff $e x(E)=1$ for any finite dimensional subspace $E \subset A$. We will use this in the following manner: let $I$ and $B$ be as above. Fix $\varepsilon>0$. Then, for any $t \in A \otimes(B / I)$ (algebraic tensor product) there is a lifting $\widehat{t} \in A \otimes B$ (again algebraic tensor product) with $\|\widehat{t}\|_{\min } \leq(1+\varepsilon)\|t\|_{\min }$. See [P2] (or [P1, ER1]) for details.

Remark 1.10. We draw the reader's attention to the fact that, if $A$ is arbitrary, since $A \otimes_{\min }(B / I)$ is a quotient $C^{*}$-algebra of $A \otimes_{\min } B$, there always exist a lifting of $t$ (say $\widehat{t}$ with $\left.\|\widehat{t}\|_{\min } \leq(1+\varepsilon)\|t\|_{\min }\right)$ in the completed tensor product $A \otimes_{\min } B$. But this seems to be of little use for us in the sequel. Indeed, it is always true that $t$ admits a lifting $\widehat{t} \in A \otimes_{\max } B$ with $\|\widehat{t}\|_{\max } \leq(1+\varepsilon)\|t\|_{\min }$, but in general we cannot derive from this that $\|t\|_{\max } \leq(1+\varepsilon)\|t\|_{\min }$, unless we know that $\widehat{t}$ is in the algebraic tensor product, in which case we $d o$ obtain $\|t\|_{\max } \leq(1+\varepsilon)\|t\|_{\min }$. The crucial point is that, in general, the 
canonical map $A \otimes_{\max } B \rightarrow A \otimes_{\min } B$ is not injective unless restricted to the algebraic tensor product.

\section{$\S 2$. Proofs}

We will use exactness in the same way as in [P3] and [JP], through the following result which is implicit in [JP].

Lemma 2.1. Let $E, F$ be exact operator spaces and let $C=\operatorname{ex}(E) \operatorname{ex}(F)$. Let $A_{1}, A_{2}$ be two $C^{*}$-algebras such that either $A_{1}$ or $A_{2}$ is $Q W E P$. Let $u: E \times F \rightarrow B(\mathcal{H})$ be a j.c.b. bilinear map. Then for any finite sequences $\left(a_{i}\right),\left(b_{j}\right),\left(x_{i}\right),\left(y_{j}\right)$ in $E, F, A_{1}, A_{2}$ respectively we have

$\left\|\sum u\left(a_{i}, b_{j}\right) \otimes x_{i} \otimes y_{j}\right\|_{B(\mathcal{H}) \otimes_{\min }\left(A_{1} \otimes_{\max } A_{2}\right)} \leq C\left\|\sum a_{i} \otimes x_{i}\right\|_{E \otimes_{\min } A_{1}}\left\|\sum b_{j} \otimes y_{j}\right\|_{F \otimes_{\min } A_{2}}$.

Equivalently, $u$ "extends" to a bounded bilinear form from $E \otimes_{\min } A_{1} \times F \otimes_{\min } A_{2}$ to $B(\mathcal{H}) \otimes_{\min }\left(A_{1} \otimes_{\max } A_{2}\right)$, taking $\left(e \otimes a_{1}, f \otimes a_{2}\right)$ to $u(e, f) \otimes a_{1} \otimes a_{2}$.

Proof. We may assume that $A_{1}$ is QWEP, so that $A_{1} \simeq B_{1} / I_{1}$ with $B_{1}$ WEP. Taking for $B_{2}$ the full $C^{*}$-algebra of a suitably large free group we may assume $A_{2} \simeq B_{2} / I_{2}$. We denote by $q_{i}: B_{i} \rightarrow A_{i}$ the quotient map. We will use the isometric identity

$$
B_{1} \otimes_{\min } B_{2} \simeq B_{1} \otimes_{\max } B_{2}
$$

This is due to Kirchberg [Ki2], (see [P2] for a simpler proof). In particular we have

$$
\left\|q_{1} \otimes q_{2}: B_{1} \otimes_{\min } B_{2} \rightarrow A_{1} \otimes_{\max } A_{2}\right\|_{c b} \leq 1
$$

hence

$$
\left\|I_{B \mathcal{H})} \otimes q_{1} \otimes q_{2}: B(\mathcal{H}) \otimes_{\min }\left(B_{1} \otimes_{\min } B_{2}\right) \rightarrow B(\mathcal{H}) \otimes_{\min }\left(A_{1} \otimes_{\max } A_{2}\right)\right\| \leq 1
$$

We may as well assume, without loss of generality, that $E$ and $F$ are finite dimensional. We now use the exactness of $E$ and $F$ (see (1.9)): let $C_{1}=e x(E)$ and $C_{2}=e x(F)$, assuming

$$
\left\|\sum a_{i} \otimes x_{i}\right\|_{\min }<1 \quad \text { and } \quad\left\|\sum b_{j} \otimes y_{j}\right\|_{\min }<1
$$


we can find elements $t_{1} \in E \otimes B_{1}$ and $t_{2} \in F \otimes B_{2}$ with $\left\|t_{1}\right\|_{\min }<C_{1},\left\|t_{2}\right\|_{\min }<C_{2}$ such that $\left(I \otimes q_{1}\right)\left(t_{1}\right)=\sum a_{i} \otimes x_{i}$ and $\left(I \otimes q_{2}\right)\left(t_{2}\right)=\sum b_{j} \otimes x_{j}$. By $(1.1)^{\prime}$ we have

$$
\left\|(u)_{B_{1}, B_{2}}\left(t_{1}, t_{2}\right)\right\| \leq\|u\|_{j c b}\left\|t_{1}\right\|_{\min }\left\|t_{2}\right\|_{\min }
$$

and moreover $(u)_{B_{1}, B_{2}}\left(t_{1}, t_{2}\right)$ lies in the algebraic tensor product $B(\mathcal{H}) \otimes B_{1} \otimes B_{2}$. But clearly

$$
\left(I \otimes q_{1} \otimes q_{2}\right)(u)_{B_{1}, B_{2}}=(u)_{A_{1}, A_{2}} \circ\left(I \otimes q_{1}, I \otimes q_{2}\right)
$$

hence by (2.2)' (and Remark 1.10)

$$
\left\|\sum u\left(a_{i}, b_{j}\right) \otimes x_{i} \otimes y_{j}\right\|_{B(\mathcal{H}) \otimes_{\min }\left(A_{1} \otimes_{\max } A_{2}\right)} \leq C_{1} C_{2}\|u\|_{j c b}
$$

By homogeneity, this completes the proof.

Lemma 2.2. Let $A, B$ be arbitrary $C^{*}$-algebras and let $u: A \times B \rightarrow \mathbb{C}$ be a j.c.b. bilinear map of finite rank with $\|u\|_{j c b} \leq 1$. Then $u$ satisfies the conclusion of Lemma 2.1 with $C=1$.

Proof. The same argument as above for Lemma 2.1 can be used to establish this, but the exactness of $E$ and $F$ has to be replaced by the following known fact: Let $E \subset A$ be a finite dimensional subspace and let $Z$ be a finite dimensional operator space. Then for any linear map $\alpha: A \rightarrow Z$ with $\|\alpha\|_{c b}<1$, the restriction $\alpha_{\mid E}: E \rightarrow Z$ is 1-exact in the following sense: there is an integer $N$, a subspace $G \subset M_{N}$ and a factorization $E \stackrel{v}{\longrightarrow} G \stackrel{w}{\longrightarrow} Z$ of $\alpha_{\mid E}$ with $\|v\|_{c b}\|w\|_{c b}<1$.

This was observed in [P1]. Here is a quick sketch of proof: let $I \subset B$ be an ideal in a $C^{*}$ algebra $B$ and let $q: B \rightarrow B / I$ be the quotient map. For any operator space $Z$ we denote by $q_{Z}: Z \otimes_{\min } B \rightarrow Z \otimes_{\min } B / I$ the (contractive) map associated to $I_{Z} \otimes q$. Moreover we let

$$
Q(Z)=\left(Z \otimes_{\min } B\right) /\left(Z \otimes_{\min } I\right) \quad \text { and } \quad R(Z)=Z \otimes_{\min }(B / I) .
$$

It suffices to show (and this is precisely what we use to prove Lemma 2.2) that $a_{\mid E}: E \rightarrow Z$ naturally induces a mapping $\widehat{\alpha}: R(E) \rightarrow Q(Z)$ with $\|\widehat{\alpha}\|<1$. To verify this, note that since $q_{A}$ is a $*$-homomorphism, it induces an isometric isomorphism

$$
R(A) \simeq A \otimes_{\min } B / \operatorname{ker}\left(q_{A}\right)
$$


But since $\alpha$ has finite rank, it is easy to see that the map $\alpha \otimes q: A \otimes_{\min } B \rightarrow Z \otimes_{\min }(B / I)$ (obtained by extension from $\alpha \otimes q$ ) must vanish on $\operatorname{ker}\left(q_{A}\right)$, hence by (2.3) it defines a map $\widetilde{\alpha}: R(A) \rightarrow Q(Z)$ with $\|\widetilde{\alpha}\| \leq\|\alpha \otimes q\| \leq\|\alpha\|_{c b}<1$. Finally since $\widehat{\alpha}: R(E) \rightarrow Q(Z)$ is but the restriction of $\widetilde{\alpha}$ to $R(E)$ we obtain $\|\widehat{\alpha}\| \leq\|\alpha\|_{c b}<1$.

Using this fact we complete the proof as follows. Assume for simplicity that $\|u\|_{j c b}<1$. Since $u$ is assumed of finite rank, there is a finite dimensional operator space $Z$ and linear maps $\alpha: A \rightarrow Z$ and $\beta: B \rightarrow Z^{*}$ with $\|\alpha\|_{c b} \leq 1$ and $\|\beta\|_{c b} \leq 1$, such that

$$
u(a, b)=\langle\alpha(a), \beta(b)\rangle
$$

Let $E \subset A$ and $F \subset B$ be finite dimensional subspaces (we can take $E=\operatorname{span}\left(a_{i}\right)$ and $\left.F=\operatorname{span}\left(b_{j}\right)\right)$. Then the maps $\alpha_{\mid E}: E \rightarrow Z$ and $\beta_{\mid F}: F \rightarrow Z^{*}$ are 1-exact in the above sense. The rest of the proof is then identical to that of Lemma 2.1.

Proposition 2.3. Let $A$ be a $C^{*}$-algebra and let $F$ be an exact operator space. Then any j.c.b. bilinear form $u: A \times F \rightarrow \mathbb{C}$ with $\|u\|_{j c b} \leq 1$ satisfies the conclusion of Lemma 2.1 with $C=e x(F)$.

Proof. Indeed, we can run the same argument as for Lemma 2.1, but using the remarks at the end of $\S 1.9$. With the same notation as in Lemma 2.1 (but here $E=A$ and $C_{1}=1$ ) we can find $t_{1}$ in the completed tensor product $A \otimes_{\min } B_{1}$ satisfying all the properties in the proof of Lemma 2.1, but since $t_{2}$ can still be chosen in the algebraic tensor product $F \otimes B_{2}$, we find again $(u)_{B_{1}, B_{2}}\left(t_{1}, t_{2}\right)$ in the algebraic tensor product $B_{1} \otimes B_{2}$, hence we can complete the proof as for Lemma 2.1.

In the previous versions of GT, the crucial ingredient is always the existence of a "special" realization of Hilbert space as a function space of some sort. The analog of this in our situation is the span of a free family of "generalized circular elements" in the sense of [S1]. This is an extension of Voiculescu's circular systems (see [VDN]), as follows.

Let $H$ be any Hilbert space. Let $H^{\otimes n}=H \otimes_{2} \cdots \otimes_{2} H$ ( $n$ times). We denote by $\mathcal{F}(H)$ the full Fock space over $H$, i.e.

$$
\mathcal{F}(H)=\mathbb{C} \oplus H \oplus H^{\otimes 2} \oplus \cdots \oplus H^{\otimes n} \oplus \cdots
$$


We denote by $\Omega$ the unit in $\mathbb{C}$ view as an element of $\mathcal{F}(H)$. For any $h$ in $H$, we denote by $\ell(h): \mathcal{F}(H) \rightarrow \mathcal{F}(H)$ (resp. $r(h): \mathcal{F}(H) \rightarrow \mathcal{F}(H)$ ) the left (resp. right) creation operator, defined by: $\ell(h) \Omega=h$ (resp. $r(h) \Omega=h$ ) and for any $x$ in $H^{\otimes n}$ with $n>0: \ell(h) x=h \otimes x$ (resp. $r(h) x=x \otimes h$ ). We will assume that $H$ admits an orthonormal basis which can be split in two parts with equal cardinality,

$$
\left\{e_{i} \mid i \in I\right\} \quad \text { and } \quad\left\{e_{i}^{\prime} \mid i \in I\right\}
$$

so that the union $\left\{e_{i} \mid i \in I\right\} \cup\left\{e_{i}^{\prime} \mid i \in I\right\}$ is an orthonormal system. We will denote

$$
\begin{aligned}
& \ell_{i}=\ell\left(e_{i}\right) \quad \text { and } \quad r_{i}=r\left(e_{i}\right) \\
& \ell_{i}^{\prime}=\ell\left(e_{i}^{\prime}\right) \quad \text { and } \quad r_{i}^{\prime}=r\left(e_{i}^{\prime}\right)
\end{aligned}
$$

Then we define, for any $\lambda>0$, the "generalized circular elements" as follows:

$$
c_{i}(\lambda)=\lambda^{1 / 2} \ell_{i}+\lambda^{-1 / 2} \ell_{i}^{\prime *}
$$

and

$$
d_{i}(\lambda)=\lambda^{1 / 2} r_{i}^{\prime}+\lambda^{-1 / 2} r_{i}^{*}
$$

The von Neumann algebra generated by such systems is studied in [S1]. (When $\lambda=1$, we recover circular elements in Voiculescu's sense.)

We will use the following basic properties of these operators (the key point is the second one):

Lemma 2.4. Let $\lambda_{i}>0$ be fixed $(i \in I)$. Let $x_{i}=c_{i}\left(\lambda_{i}\right)$ and $y_{i}=d_{i}\left(\lambda_{i}\right)$.

(i) Let $E, F$ be operator spaces and let $\left(a_{i}, b_{i}\right)_{i \in I}$ be a finitely supported family in $E \times F$. Then we have

$$
\begin{aligned}
\left\|\sum a_{i} \otimes x_{i}\right\| & \leq\left\|\sum \lambda_{i} a_{i}^{*} a_{i}\right\|^{1 / 2}+\left\|\sum \lambda_{i}^{-1} a_{i} a_{i}^{*}\right\|^{1 / 2} \\
\left\|\sum b_{i} \otimes y_{i}\right\| & \leq\left\|\sum \lambda_{i} b_{i}^{*} b_{i}\right\|^{1 / 2}+\left\|\sum \lambda_{i}^{-1} b_{i} b_{i}^{*}\right\|^{1 / 2} .
\end{aligned}
$$

(ii) The families $\left\{x_{i}\right\}\left\{y_{j}\right\}$ "double commute" which means: $x_{i} y_{j}=y_{j} x_{i}$ and $x_{i}^{*} y_{j}=y_{j} x_{i}^{*}$ for all $i, j$ in $I$. 
(iii) $\left\langle x_{i} y_{i} \Omega, \Omega\right\rangle=1$ and $\left\langle x_{i} y_{j} \Omega, \Omega\right\rangle=0$ for all $i \neq j$.

Proof. To prove (i) note that each $\left\{\ell_{i}\right\},\left\{\ell_{i}^{\prime}\right\},\left\{r_{i}\right\}$ or $\left\{r_{i}^{\prime}\right\}$ is a family of isometries with orthogonal ranges and if $\left(s_{i}\right)$ is any such family we must have $\left\|\sum s_{i} s_{i}^{*}\right\| \leq 1$. By an easy and well known consequence of Cauchy-Schwarz, we have for any finitely supported family of operators $\left(s_{i}, t_{i}\right)_{i \in I}$

$$
\left\|\sum s_{i} \otimes t_{i}\right\| \leq\left\|\sum s_{i} s_{i}^{*}\right\|^{1 / 2}\left\|\sum t_{i}^{*} t_{i}\right\|^{1 / 2}
$$

and of course also

$$
\leq\left\|\sum s_{i}^{*} s_{i}\right\|^{1 / 2}\left\|\sum t_{i} t_{i}^{*}\right\|^{1 / 2}
$$

Thus by the triangle inequality we have

$$
\begin{aligned}
\left\|\sum a_{i} \otimes x_{i}\right\| & \leq\left\|\sum a_{i} \lambda_{i}^{1 / 2} \otimes \ell_{i}\right\|+\left\|\sum a_{i} \lambda_{i}^{-1 / 2} \otimes \ell_{i}^{\prime *}\right\| \\
& \leq\left\|\sum a_{i}^{*} a_{i} \lambda_{i}\right\|^{1 / 2}\left\|\sum \ell_{i} \ell_{i}^{*}\right\|^{1 / 2}+\left\|\sum a_{i} a_{i}^{*} \lambda_{i}^{-1}\right\|^{1 / 2}\left\|\sum \ell_{i}^{\prime} \ell_{i}^{\prime *}\right\|^{1 / 2}
\end{aligned}
$$

which implies the first part of (i). The same argument proves the other part.

To prove (ii) note that for all $h, k$ in $H$ the operators $\ell(h)$ and $r(k)$ obviously commute and

$$
r(k)^{*} \ell(h)-\ell(h) r(k)^{*}=\langle h, k\rangle P_{\Omega} .
$$

It is then but an elementary verification to check (ii).

(iii) We have $y_{i} \Omega=\lambda_{i}^{1 / 2} e_{i}^{\prime}$ and $x_{i} e_{i}^{\prime}=\lambda_{i}^{1 / 2} e_{i} \otimes e_{i}^{\prime}+\lambda_{i}^{-1 / 2} \Omega$ hence $\left\langle x_{i} y_{i} \Omega, \Omega\right\rangle=\langle\Omega, \Omega\rangle=1$. Similarly if $i \neq j$ we find $x_{i} y_{j} \Omega=\lambda_{i}^{1 / 2} \lambda_{j}^{1 / 2} e_{i} \otimes e_{j}^{\prime}$ hence $\left\langle x_{i} y_{j} \Omega, \Omega\right\rangle=0$.

In order to be able to use the generalized circular families, it is crucial to know the following:

Lemma 2.5. The von Neumann algebra $W^{*}\left(x_{i}: i \in I\right)$ generated by the family $\left\{x_{i} \mid i \in\right.$ $I\}$ defined in the preceding lemma is QWEP.

Proof. Let $H_{\mathbb{R}}$ be a real Hilbert space of dimension $2|I|$, and denote by $f_{i}, f_{i}^{\prime}, i \in$ $I$ its orthonormal basis. Let $U_{t}: H_{\mathbb{R}} \rightarrow H_{\mathbb{R}}$ be given by $U_{t}\left(f_{i}\right)=\cos \left(\log \left(\lambda_{i}^{2}\right) t\right) f_{i}+$ $\sin \left(\log \left(\lambda_{i}^{2}\right) t\right) f_{i}^{\prime}, U_{t}\left(f_{i}^{\prime}\right)=\cos \left(\log \left(\lambda_{i}^{2} t\right)\right) f_{i}^{\prime}-\sin \left(\log \left(\lambda_{i}^{2}\right) f_{i}\right.$. Then $U_{t}$ is a one-parameter group 
of orthogonal transformations on $H_{\mathbb{R}}$. Let $M=\Gamma\left(H_{\mathbb{R}}, U_{t}\right)^{\prime \prime}$ in its GNS representation with respect to the free quasi-free state $\phi_{U}$ (see Definition 2.3 of [S1]). By [S1, Section 4], we find that $M$ can be viewed as generated by the operators $l\left(e_{i}\right)+\lambda_{i} l\left(e_{i}^{\prime}\right)^{*}$ and is therefore isomorphic to the algebra $W^{*}\left(x_{i}: i \in I\right)$. Hence it is sufficient to prove that $M$ is QWEP.

Since $U_{t}$ is almost-periodic, it follows from Theorem 6.4 and Theorem 6.7 of [S1] that

$$
\left(M, \phi_{U}\right) \cong *_{i \in I}\left\{\left(M_{2 \times 2}, \operatorname{Tr}\left(\left(\begin{array}{cc}
\frac{1}{1+\lambda^{2}} & 0 \\
0 & \frac{\lambda^{2}}{1+\lambda^{2}}
\end{array}\right)\right)\right) * L^{\infty}[0,1]\right\}
$$

Hence by the results of $\mathrm{K}$. Dykema [D], the centralizer $M^{\phi_{U}}$ of $\phi_{U}$ is isomorphic to $L\left(\mathbb{F}_{\infty}\right)$. Thus $M^{\phi_{U}}$ is a factor. Moreover, by Kirchberg's results, it is QWEP, since $L\left(\mathbb{F}_{\infty}\right)$ can be embedded into the ultrapower of the hyperfinite $\mathrm{II}_{1}$ factor (see [Ki1]).

Since $U_{t}$ is almost-periodic, it follows that the modular group of $\phi_{U}$ is almost-periodic, so that $\phi_{U}$ is an almost-periodic state (see $[\mathrm{C}]$ ). By Connes' results in $[\mathrm{C}]$, it follows from the fact that $M^{\phi_{U}}$ is a factor that

$$
M \cong\left(M^{\phi_{U}} \otimes B\left(\ell^{2}\right) \otimes B(H)\right) \rtimes G
$$

where $G$ is a discrete group (isomorphic to the multiplicative subgroup of $(0,+\infty)$ generated by the set $\left.\left\{\lambda_{i}^{2}: i \in I\right\}\right)$. Since $G$ is Abelian, hence amenable, and $M^{\phi_{U}}$ is QWEP, it follows that $M$ is QWEP (see the remark after Prop. 1.3 in [Ki1]).

Remark. It is possible to give an alternate proof that $M$ is QWEP. Indeed, it is sufficient to find a sequence of states $\phi_{i}$ on matrix algebras $A_{i}=M_{n_{i} \times n_{i}}$, a free ultrafilter $\omega$ and an embedding

$$
i: M \rightarrow \prod_{\omega}\left(A_{i}, \phi_{i}\right)
$$

so that $\left.\prod \sigma^{\phi_{i}}\right|_{i(M)}=\sigma^{\phi_{U}}$. Indeed, the latter condition implies that there exists a statepreserving conditional expectation $E: \prod_{\omega}\left(A_{i}, \phi_{i}\right)$ onto $i(M)$; since $\prod A_{i}$ is WEP, $\prod_{\omega}\left(A_{i}, \phi_{i}\right)$ is QWEP, and thus $i(M)$ is QWEP.

To construct the embedding $i$ one can utilize the model for free quasi-free states involving matrices with CAR variables as entries (described in [S2]). To make sure that the matrices involved stay bounded in norm, one must cut them off using continuous functional calculus; 
it can be verified that this procedure can be performed in a way that does not affect their joint $*$-distribution. We leave the details to the reader.

We can now prove our main results.

Proof of Theorem 0.3. Let $A_{1}$ (resp. $A_{2}$ ) be the von Neumann algebra generated by $\left(x_{i}\right)$ (resp. $\left.\left(y_{i}\right)\right)$. By Lemma 2.4 (iii)

$$
\left|\sum U\left(a_{i}, b_{i}\right)\right|=\left|\sum_{i j} U\left(a_{i}, b_{j}\right)\left\langle x_{i} y_{j} \Omega, \Omega\right\rangle\right|
$$

hence by Lemma 2.4 (ii)

$$
\leq\left\|\sum_{i j} U\left(a_{i}, b_{j}\right) \otimes x_{i} \otimes y_{j}\right\|_{B(\mathcal{H}) \otimes_{\min }\left(A_{1} \otimes_{\max } A_{2}\right)}
$$

hence by Lemma 2.1

$$
\leq C\left\|\sum a_{i} \otimes x_{i}\right\|_{\min }\left\|\sum b_{i} \otimes y_{i}\right\|_{\min }
$$

hence by Lemma 2.4 (i) we obtain (0.13). Taking $\lambda_{i}=\lambda$ for all $i$ and then choosing $\lambda^{2}=\left\|\sum b_{i} b_{i}^{*}\right\|^{1 / 2}\left\|\sum b_{i}^{*} b_{i}\right\|^{-1 / 2}$, we derive (0.14) from (0.13).

Proof of Theorem 0.4. By Theorem 0.3 and by (1.3) we have (note that the argument of $U\left(a_{i}, b_{i}\right)$ can be absorbed e.g. by $\left.a_{i}\right)$

$$
\sum\left|U\left(a_{i}, b_{i}\right)\right| \leq C[I+I I]
$$

where

$$
\begin{aligned}
I & =\left\|\sum \lambda_{i} a_{i}^{*} a_{i}\right\|+\left\|\sum \lambda_{i}^{-1} b_{i} b_{i}^{*}\right\| \\
I I & =\left\|\sum \lambda_{i}^{-1} a_{i} a_{i}^{*}\right\|+\left\|\sum \lambda_{i} b_{i}^{*} b_{i}\right\| .
\end{aligned}
$$

By a very easy adaptation of Proposition 1.3 this implies the existence of states $f_{1}, f_{2}, g_{1}, g_{2}$ on $A$ and $B$ such that for any $(a, b)$ in $E \times F$ and any $\lambda>0$ (note it is crucial that the states do not depend on $\lambda$ !)

$$
|U(a, b)| \leq C\left[\lambda f_{1}\left(a^{*} a\right)+\lambda^{-1} g_{1}\left(b b^{*}\right)+\lambda^{-1} f_{2}\left(a a^{*}\right)+\lambda g_{2}\left(b^{*} b\right)\right] .
$$

Applying this to $\left(t a, t^{-1} b\right)$ instead of $(a, b)$, and taking the infimum over $t>0$ we obtain by $(1.3)$

$$
\begin{aligned}
|U(a, b)| & \leq 2 C\left(\lambda f_{1}\left(a^{*} a\right)+\lambda^{-1} f_{2}\left(a a^{*}\right)\right)^{1 / 2}\left(\lambda^{-1} g_{1}\left(b b^{*}\right)+\lambda g_{2}\left(b^{*} b\right)\right)^{1 / 2} \\
& \leq 2 C\left(f_{1}\left(a^{*} a\right) g_{1}\left(b b^{*}\right)+f_{2}\left(a a^{*}\right) g_{2}\left(b^{*} b\right)+R_{\lambda}\right)^{1 / 2}
\end{aligned}
$$


where

$$
R_{\lambda}=\lambda^{2} f_{1}\left(a^{*} a\right) g_{2}\left(b^{*} b\right)+\lambda^{-2} f_{2}\left(a a^{*}\right) g_{1}\left(b b^{*}\right) .
$$

By (1.3) we have

$$
\inf _{\lambda>0} R_{\lambda}=2\left(f_{1}\left(a^{*} a\right) g_{2}\left(b^{*} b\right) f_{2}\left(a a^{*}\right) g_{1}\left(b b^{*}\right)\right)^{1 / 2}
$$

hence by (1.3) again (with a different grouping of terms

$$
\leq f_{1}\left(a^{*} a\right) g_{1}\left(b b^{*}\right)+f_{2}\left(a a^{*}\right) g_{2}\left(b^{*} b\right)
$$

thus we obtain

$$
|U(a, b)| \leq 2 \sqrt{2} C\left(f_{1}\left(a^{*} a\right) g_{1}\left(b b^{*}\right)+f_{2}\left(a a^{*}\right) g_{2}\left(b^{*} b\right)\right)^{1 / 2}
$$

which implies (0.15). Clearly, (0.15) implies (0.16) by Cauchy-Schwarz.

Now, by Proposition 1.7, (0.16) can be reformulated as follows: for any $w$ in $E \otimes F$ we have

$$
|\langle U, w\rangle| \leq K\left[\|w\|_{h}+\left\|{ }^{t} w\right\|_{h}\right]
$$

Thus $U$ defines a continuous linear form with norm $\leq K$ on the subspace

$$
\left\{\left(w,{ }^{t} w\right) \mid w \in E \otimes F\right\} \subset\left(E \otimes_{h} F\right) \oplus_{1}\left(F \otimes_{h} E\right)
$$

equipped with the norm $\|(x, y)\|=\|x\|_{h}+\|y\|_{h}$. By the Hahn-Banach theorem, there are linear forms $\varphi_{1} \in\left(E \otimes_{h} F\right)^{*}$ and $\varphi_{2} \in\left(F \otimes_{h} E\right)^{*}$ with $\max \left\{\left\|\varphi_{1}\right\|,\left\|\varphi_{2}\right\|\right\} \leq K$ such that

$\forall w \in E \otimes F \quad\langle U, w\rangle=\varphi_{1}(w)+\varphi_{2}\left({ }^{t} w\right)$.

Let $u\left(\operatorname{resp} .{ }^{t} v\right)$ be the bilinear forms associated to $\varphi_{1}$ (resp. $\varphi_{2}$ ). Going back to (0.5), we see that $\left\|\varphi_{1}\right\| \leq K$ (resp. $\left.\left\|\varphi_{2}\right\| \leq K\right)$ is equivalent to $\|u\|_{c b} \leq K,\left(\operatorname{resp} .\left\|{ }^{t} v\right\|_{c b} \leq K\right)$. Clearly $U=u+v$. Thus the final assertion follows.

Proof of Theorem 0.5. This clearly reduces to the finite rank case. The proof is then the same as for Theorem 0.4 but using Lemma 2.2 instead of Lemma 2.1.

Proof of Corollary 0.6. Let $\varphi_{1}, \varphi_{2}$ be as in the above proof of Theorem 0.4. Now since $\otimes_{h}$ is an injective tensor product ( $c f$. [ER1]), $\varphi_{1}$ and $\varphi_{2}$ can be extended to linear forms

$$
\Phi_{1}: A \otimes_{h} B \rightarrow \mathbb{C} \text { and } \Phi_{2}: B \otimes_{h} A \rightarrow \mathbb{C}
$$


such that $\left\|\Phi_{i}\right\|=\left\|\varphi_{i}\right\|,(i=1,2)$. Let $U_{i}: A \times B \rightarrow \mathbb{C}$ be the bilinear forms associated to $\Phi_{i} \quad(i=1,2)$. By $(0.8)$ and $(1.4)$, we have

$$
\begin{aligned}
\left\|U_{1}+U_{2}\right\|_{j c b} & \leq\left\|\Phi_{1}\right\|+\left\|\Phi_{2}\right\| \\
& \leq 4 \sqrt{2} C .
\end{aligned}
$$

Thus if we set $\widehat{U}=U_{1}+U_{2}$, we obtain Corollary 0.6 .

Proof of Corollary 0.7. Let $u$ be the bilinear form associated to $T$, so that $T=\widetilde{u}$. By the proof of Corollary 0.6, we have a decomposition

$$
u=u_{1}+u_{2}
$$

with

$$
\max \left(\left\|u_{1}\right\|_{c b},\left\|^{t} u_{2}\right\|_{c b}\right) \leq 2 \sqrt{2} C\|u\|_{j c b}
$$

By the results recalled in 1.4, the linear map $\widetilde{u}_{1}: E \rightarrow F^{*}\left(\operatorname{resp} . \widetilde{u}_{2}: E \rightarrow F^{*}\right)$ can be factorized through $H_{r}$ (resp. $H_{c}$ ) for some $H$. More precisely, we have factorizations

$$
\begin{aligned}
& \widetilde{u}_{1}: E \stackrel{\alpha_{1}}{\longrightarrow} H_{r} \stackrel{\beta_{1}}{\longrightarrow} F^{*} \\
& \widetilde{u}_{2}: E \stackrel{\alpha_{2}}{\longrightarrow} H_{c} \stackrel{\beta_{2}}{\longrightarrow} F^{*}
\end{aligned}
$$

with

$$
\max \left\{\left\|\alpha_{1}\right\|_{c b}\left\|\beta_{1}\right\|_{c b},\left\|\alpha_{2}\right\|_{c b}\left\|\beta_{2}\right\|_{c b}\right\} \leq 2 \sqrt{2} C\|u\|_{j c b}
$$

By homogeneity, we can adjust $\alpha_{i}, \beta_{i}$ so that

$$
\left\|\alpha_{1}\right\|_{c b}=\left\|\beta_{1}\right\|_{c b} \quad \text { and } \quad\left\|\alpha_{2}\right\|_{c b}=\left\|\beta_{2}\right\|_{c b}
$$

Then, if we define

$$
v: E \rightarrow H_{r} \oplus H_{c} \quad \text { and } \quad w: H_{r} \oplus H_{c} \rightarrow F^{*}
$$

by

$$
v(e)=\alpha_{1}(e) \oplus \alpha_{2}(e) \text { and } \quad w(x \oplus y)=\beta_{1}(x)+\beta_{2}(y)
$$

we obtain $\widetilde{u}=w v$ and

$$
\begin{aligned}
\|w\|_{c b}\|v\|_{c b} & \leq \max \left\{\left\|\alpha_{1}\right\|_{c b},\left\|\alpha_{2}\right\|_{c b}\right\} \max \left\{\left\|\beta_{1}\right\|_{c b},\left\|\beta_{2}\right\|_{c b}\right\} \\
& \leq 2 \sqrt{2} C\|u\|_{j c b} .
\end{aligned}
$$


Proof of Corollary 0.8. We prove this using duality. By (0.16) and by Proposition 1.7 for any $w$ in $E \otimes F$ and any $U$ as in (0.16) we have

$$
\begin{aligned}
|\langle w, U\rangle| & \leq 2^{3 / 2} C\left[\|w\|_{h}+\left\|{ }^{t} w\right\|_{h}\right] \\
& \leq 2^{5 / 2} C \max \left\{\|w\|_{h},\left\|{ }^{t} w\right\|_{h}\right\}
\end{aligned}
$$

hence by $(0.11)$

$$
\|w\|_{\wedge}=\sup \left\{|\langle w, U\rangle|\|U\|_{j c b} \leq 1\right\} \leq 2^{5 / 2} C \max \left\{\|w\|_{h},\left\|{ }^{t} w\right\|_{h}\right\},
$$

which establishes the first point. The same argument allows us to deduce the second point from Theorem 0.5. Finally, the third point also follows from Theorem 0.5 using the fact that $A^{*} \otimes_{\min } B^{*}$ can be identifed with the closure of the finite rank maps in $C B\left(A, B^{*}\right)$.

Remark 2.6. By Remark 2.3, if either $A$ or $B$ is exact, the conclusions of Theorem 0.5 and Corollary 0.7 are valid without any approximability assumption. We do not know whether they are valid in full generality. Note however that, by our results, an operator $T: A \rightarrow B^{*}$ factors through a space of the form $H_{r} \oplus K_{c}$ iff it is approximable pointwise by a net of finite rank maps unifomly bounded in $C B\left(A, B^{*}\right)$. Indeed, since the identity of $H_{r} \oplus K_{c}$ is obviously approximable in this way, the only if part also holds. Thus, if Theorem 0.5 holds without any approximability assumption, this means that any $T$ in $C B\left(A, B^{*}\right)$ is approximable pointwise by a unifomly bounded net of finite rank maps.

\section{$\S 3$. Some applications}

In classical Banach space theory, GT has several well known consequences (see e.g. [P5]). Our result "automatically" allows to transfer some of these to the operator space setting. For instance, let $E$ be a Banach space. It is known that $E$ and its dual $E^{*}$ both embed in an $L_{1}$-space iff $E$ is isomorphic to a Hilbert space. Actually, this remains true if we replace " $L_{1}$-space" by "non-commutative $L_{1}$-space" (see [P5]). In the operator space case, we could not obtain an analogous characterization, but the next result essentially reduces the problem to the class of subspaces of quotients of $H_{r} \oplus K_{c}$. Recall that an operator 
space $E$ is said to have the CBAP (for completely bounded aproximation property) if the identity of $E$ is the pointwise limit of a net of finite rank maps, with uniformly bounded c.b. norms.

Corollary 3.1. Let $E$ be an operator space such that:

(i) $E$ and $E^{*}$ both embed completely isomorphically in a non-commutative $L_{1}$-space (meaning the predual of a von Neumann algebra),

(ii) $E$ has the $C B A P$, then $E$ is completely isomorphic to a quotient of a subspace of $H_{r} \oplus K_{c}$ for some Hilbert spaces $H, K$.

Proof. Note that by the Banach space result just recalled, $E$ must be isomorphic to a Hilbert space (in particular it is reflexive). Let $A, B$ be $C^{*}$-algebras. Let $J_{1}: E^{*} \rightarrow A^{*}$ and $J_{2}: E \rightarrow B^{*}$ be completely isomorphic embeddings. Let $Q_{1}: A \rightarrow E$ be the adjoint of $J_{1}$. Then let $T=J_{2} Q_{1}: A \rightarrow B^{*}$. By Corollary 0.7, $T$ factors through a space $X$ of the form $X=H_{r} \oplus K_{c}$ for suitable $H, K$. Going back to $E$, we easily deduce from this that $E$ is completely isomorphic to a quotient of a subspace of $X$.

Remark. In general, we do not know whether the assumption (ii) is needed. Note however that if either $E$ or $E^{*}$ is exact, then using Remark 2.3, (ii) can be dispensed with.

Remark. Let $E$ be the closed span of the classical Rademacher functions in $L_{1}([0,1], d t)$. By a result due to Lust-Piquard and the first author (see [P6, p. 107]), the dual $E^{*}$ is completely isomorphic to a subspace of $R \oplus C$, namely to the closed span in $R \oplus C$ of $\left\{e_{1 i} \oplus e_{i 1} \mid i \geq 1\right\}$. Let us denote by $S_{1}$ the predual of $B\left(\ell_{2}\right)$. Note that $R$ and $C$ both embed in $S_{1}$ ( $R$ can be identified naturally with the columns in $S_{1}$, and $C$ with the rows, in a suitable duality between $S_{1}$ and $B\left(\ell_{2}\right)$.) Thus, $E$ is a good illustration of the preceding corollary. In this case we have $E \subset L_{1}([0,1], d t)$ and $E^{*} \subset S_{1} \oplus S_{1}$ where $S_{1}$ is the predual of $B\left(\ell_{2}\right)$.

Another illustration is provided more generally by the family $\left\{x_{i} \mid i \in I\right\}$ appearing in Lemma 2.4: assuming for simplicity that $\lambda_{i}=\lambda>0$ does not depend on $i$, let $E_{\lambda}$ denote the weak-* closure of $\left\{x_{i} \mid i \in I\right\}$ in $B(\mathcal{F}(H))$. Note that again $E_{\lambda}$ embeds into $R \oplus C \subset S_{1} \oplus S_{1}$ 
(by Lemma $2.4(\mathrm{i})$ ) and moreover one can show there is a completely bounded projection from $W^{*}\left(x_{i}: i \in I\right)$ onto $E_{\lambda}$. Hence $E_{\lambda}{ }^{*}$ also embeds in a non-commutative $L_{1}$-space.

Remark. The preceding topic is also of interest in the isometric case. Indeed, by [Sc] there are examples of finite dimensional Banach spaces $E$, not isometric to Hilbert spaces, but such that both $E$ and $E^{*}$ embed isometrically into $L_{1}$. Curiously, however, this is known in the real case only. The complex case apparently remains open, as well as the infinite dimensional one (either real or complex). Analogously, we do not have any satisfactory completely isometric version of Corollary 3.1.

The next result gives a characterization of the operator spaces $E$ such that both $E$ and $E^{*}$ are exact (see $[\mathrm{OP}]$ for a different characterization of the same class of spaces).

Corollary 3.2. An operator space $E$ is exact as well as its (operator space) dual $E^{*}$ iff $E$ is completely isomorphic to $H_{r} \oplus K_{c}$ for some Hilbert spaces $H, K$.

Proof. By Corollary 0.7 applied to the identity operator on $E$, if $E$ and $E^{*}$ are exact, then $E$ must be completely isomorphic to a "completely complemented" subspace $F \subset H_{r} \oplus K_{c}$, meaning there is a c.b. projection $P$ from $H_{r} \oplus K_{c}$ onto $F$. By [O] this implies that $E$ is completely isomorphic to $S \oplus T$ for subspaces $S \subset H_{r}, T \subset K_{c}$. Replacing $H_{r}, K_{c}$ by these subspaces, we obtain the only if part. The converse is obvious since $H_{r}, K_{c}$ are both exact and (see e.g. [ER3]) $H_{r}^{*}=H_{c}, K_{c}^{*}=K_{r}$.

Remark. The completely isometric analog of the preceding characterization is not known: The only known spaces $E$ such that $e x(E)=e x\left(E^{*}\right)=1$ are $\ell_{\infty}^{2}, \ell_{1}^{2}, \mathbb{C} \oplus_{\infty} R_{n}, \mathbb{C} \oplus_{\infty} C_{n}$ as well as their duals, namely $\mathbb{C} \oplus_{1} C_{n}$ and $\mathbb{C} \oplus_{1} R_{n}$ (we refer to [P1] for a proof that these are exact with constant 1$)$ and $R_{n}, C_{n}(n \geq 0)$. Are these the only possible examples ?

Let $E \subset A$ be an operator subspace of a $C^{*}$-algebra. We will say that $E$ is completely complemented if there is a c.b. projection $P: A \rightarrow E$. We have then:

Corollary 3.3. Let $A, B$ be $C^{*}$-algebras. Assume that $E \subset A, E^{*} \subset B$ (completely isometrically) and that both subspaces are completely complemented. If either $A$ or $B$ is exact or if $E$ has the CBAP, then $E$ must be completely isomorphic to $H_{r} \oplus K_{c}$ for some Hilbert spaces $H, K$. 
Proof. Our assumption implies that the identity on $E$ admits a factorization of the form

$$
E \rightarrow A \rightarrow E \rightarrow B^{*} \rightarrow E
$$

hence, by Theorem 0.5, it factors through $H_{r} \oplus K_{c}$ and we conclude as in the preceding corollary using $[\mathrm{O}]$.

We now give an application to the operator Hilbert space $O H(I)$ introduced in [P7], to which we refer for more information.

Corollary 3.4. Let $A$ be a $C^{*}$-algebra, let $E \subset A$ be an exact operator space and let $I$ be an arbitrary set. If a linear map $u: E \rightarrow O H(I)$ is c.b. then there is a constant $K$ and a state $f$ on $A$ such that

$$
\forall x \in E \quad\|u(x)\|^{2} \leq K^{2}\left(f\left(x x^{*}\right) f\left(x^{*} x\right)\right)^{1 / 2} .
$$

Conversely, for any E, any map satisfying (3.1) is c.b. More precisely, we have the estimates

$$
K \leq 2^{9 / 4} e x(E)\|u\|_{c b} \quad \text { and } \quad\|u\|_{c b} \leq K .
$$

Moreover, when $E=A$, the assumption that $E$ is exact is not needed, and we have the estimates

$$
K \leq 2^{9 / 4}\|u\|_{c b} \quad \text { and } \quad\|u\|_{c b} \leq K .
$$

Proof. By definition of $O H(I), u$ is c.b. iff the mapping ${ }^{\bar{t} u} u: E \rightarrow \overline{E^{*}}$ is c.b., and we have (see [P7, p. 41])

$$
\|\bar{t} u u\|_{c b}=\|u\|_{c b}^{2} .
$$

Therefore, by Theorem 0.4 , there are states $f_{1}, f_{2}, g_{1}, g_{2}$ on $A$ such that

$\forall x \in E \quad\|u(x)\|^{2} \leq 2^{3 / 2} e x(E)^{2}\|u\|_{c b}^{2}\left[\left(f_{1}\left(x x^{*}\right) g_{1}\left(x^{*} x\right)\right)^{1 / 2}+\left(f_{2}\left(x^{*} x\right) g_{2}\left(x x^{*}\right)\right)^{1 / 2}\right]$.

Hence if we let (say) $f=4^{-1}\left(f_{1}+g_{1}+f_{2}+g_{2}\right)$ we obtain

$\forall x \in C \quad\|u(x)\|^{2} \leq 2^{9 / 2} e x(E)^{2}\|u\|_{c b}^{2}\left(f\left(x x^{*}\right) f\left(x^{*} x\right)\right)^{1 / 2}$. 
Thus we obtain the conclusion with $K^{2} \leq 2^{9 / 2} e x(E)^{2}\|u\|_{c b}^{2}$.

Conversely, if (3.1) holds then we have, by Cauchy-Schwarz, for any $x, y$ in $E$

$$
|\langle u(x), u(y)\rangle| \leq\|u(x)\|\|u(y)\| \leq K^{2}\left(f\left(x x^{*}\right) f\left(x^{*} x\right) f\left(y y^{*}\right) f\left(y^{*} y\right)\right)^{1 / 4}
$$

hence by (1.3)

$$
|\langle\bar{t} u u(x), y\rangle| \leq 2^{-1} K^{2}\left(f\left(x x^{*}\right)^{1 / 2} f\left(y^{*} y\right)^{1 / 2}+f\left(x^{*} x\right)^{1 / 2} f\left(y y^{*}\right)^{1 / 2}\right) .
$$

By the final assertion in Theorem 0.4 and by (0.8), this implies $\|\bar{t} u u\|_{c b} \leq K^{2}$, and by (3.2) we obtain $\|u\|_{c b} \leq K$.

Now if $E=A$, we may use (ii) in Theorem 0.5 (since that $O H(I)$ has the CBAP) to justify the last assertion.

Remark. The first part of Corollary 3.4 may fail if we do not assume $E$ exact: for instance if $u$ is the identity on $O H$ and if $T_{i}$ is an orthonormal basis of $O H$ formed of selfadjoint operators (see [P7] p. 19), we have $\sum_{1}^{n}\left\|T_{i}\right\|^{2}=n$ and $\sum_{1}^{n}\left(f\left(T_{i} T_{i}^{*}\right) f\left(T_{i}^{*} T_{i}\right)\right)^{1 / 2}=$ $f\left(\sum_{1}^{n} T_{i}^{2}\right) \leq\left\|\sum_{1}^{n} T_{i}^{2}\right\|=n^{1 / 2}$.

The next statement improves an unpublished result of Marius Junge (see $[\mathrm{J}]$ ) who proved (3.3) (say assuming $u$ is a complete contraction) with $(\log (n))^{2}$ instead of $\log (n)$. Junge's proof already used tools from interpolation theory similar to the ones we use below.

Corollary 3.5. Assume $A=B(H)$. Let $u: A \rightarrow O H(I)$ be a c.b. map. Assume (3.1). Then for any $n>1$ and for any $n$-tuple $x_{1}, \ldots, x_{n}$ in $A$ we have

$$
\sum_{1}^{n}\left\|u\left(x_{i}\right)\right\|^{2} \leq K^{2}(c \log (n)+1)\left\|\sum^{n} x_{i} \otimes \bar{x}_{i}\right\|_{\min }
$$

where $c>0$ is a numerical absolute constant (independent of $n$ or $u$ ).

Remark. If $A$ is an arbitrary $C^{*}$-algebra, the same argument can be adapted (using [H2]) to show that (3.3) holds with $\left\|\sum x_{i} \otimes \bar{x}_{i}\right\|_{\max }$ instead of $\left\|\sum x_{i} \otimes \bar{x}_{i}\right\|_{\min }$.

Note: In the sequel, the constants $c_{1}, c_{2}, c_{3}, \ldots$ will all be absolute positive numerical constants bounded independently of any parameter (we can safely say they are all majorized by $\left.10^{3} !\right)$.

To prove Corollary 3.5, we will first need the following consequence of (3.1). 
Lemma 3.6. In the situation of Corollary 3.4, assume (for simplicity) that $A=M_{N}$. We identify $f$ with an $N \times N$ matrix $f \geq 0$ with unit trace so that the state $f$ is identified with the map $x \rightarrow \operatorname{tr}(f x)$. Then, if (3.1) holds, there are constants $c_{1}$ and $c_{2}$ such that for every $x$ in $E$ and for any $t \geq 2$

$$
\|u(x)\|^{2} \leq K^{2}\left[c_{1} \log (t) \operatorname{tr}\left(f^{1 / 2} x f^{1 / 2} x^{*}\right)+\frac{c_{2}}{t} f\left(x x^{*}+x^{*} x\right)\right]
$$

Proof. By a change of basis, we may clearly assume that $f$ is a diagonal matrix so that setting $\lambda_{i}=f_{i i}$ we have $\lambda_{i}>0, \sum \lambda_{i}=1$ and (3.1) becomes

$\forall x \in E \quad\|u(x)\|^{2} \leq K^{2}\left(\sum_{i j} \lambda_{i}\left|x_{i j}\right|^{2} \sum_{i j} \lambda_{j}\left|x_{i j}\right|^{2}\right)^{1 / 2}$.

Fix a number $t \geq 2$. Let $S(1)=\left\{(i, j) \mid t^{-2} \leq \lambda_{i} \lambda_{j}^{-1} \leq t^{2}\right\}, S(2)=\left\{(i, j) \mid \lambda_{i} \lambda_{j}^{-1}>t^{2}\right\}$, and $S(3)=\left\{(i, j) \mid \lambda_{i} \lambda_{j}^{-1}<t^{-2}\right\}$. Then let $u_{k}(x)=u\left(\sum_{i j \in S(k)} x_{i j} e_{i j}\right)(k=1,2,3)$ so that $u=u_{1}+u_{2}+u_{3}$. Since on $S(2)$ we have $\lambda_{j} \lambda_{i}^{-1}<t^{-2}$ and similarly on $S(3)$, we can write

$$
\left\|u_{2}(x)\right\|^{2} \leq K^{2} t^{-1} \sum_{i j} \lambda_{i}\left|x_{i j}\right|^{2}=K^{2} t^{-1} f\left(x x^{*}\right)
$$

and

$$
\left\|u_{3}(x)\right\|^{2} \leq K^{2} t^{-1} \sum_{i j} \lambda_{j}\left|x_{i j}\right|^{2}=K^{2} t^{-1} f\left(x^{*} x\right)
$$

We now turn to $u_{1}$. We will use freely the standard notation from interpolation theory as described e.g. in [BL]. Let $E_{0}$ (resp. $\left.E_{1}\right)$ be the space $E$ equipped with the norm $f\left(x^{*} x\right)^{1 / 2}$ (resp. $\left.f\left(x x^{*}\right)^{1 / 2}\right)$. We denote for simplicity $(1 \leq q<\infty)$

$$
E(1 / 2)=\left(E_{0}, E_{1}\right)_{1 / 2} \quad \text { and } \quad E(1 / 2, q)=\left(E_{0}, E_{1}\right)_{1 / 2, q}
$$

It is a well known fact that since $E_{0}, E_{1}$ are both Hilbertian we have $E(1 / 2) \simeq E(1 / 2,2)$ with equivalence constants bounded independently of $N$ or $E$. Moreover, it is a classical fact that

$$
\|x\|_{E(1 / 2)}=\operatorname{tr}\left(f^{1 / 2} x f^{1 / 2} x^{*}\right) .
$$


By the extremal property of $E(1 / 2,1)$ (see [BL, p. 58]), (3.1) implies an estimate of the form

$$
\forall x \in E \quad\|u(x)\| \leq c_{3} K\|x\|_{E(1 / 2,1)}
$$

Since for any $x$ in $\operatorname{span}\left\{e_{i j} \mid(i, j) \in S(1)\right\}$ we have $t^{-1}\|x\|_{E_{0}} \leq\|x\|_{E_{1}} \leq t\|x\|_{E_{0}}$, it follows by a well known estimate that

$$
\|x\|_{E(1 / 2,1)} \leq c_{4}(\log t)^{1 / 2}\|x\|_{E(1 / 2,2)}
$$

hence combining this last bound with (3.6), (3.7) and the equivalence $E(1 / 2) \simeq E(1 / 2,2)$ we obtain for any $x$ in $\operatorname{span}\left\{e_{i j} \mid(i, j) \in S(1)\right\}$

$$
\|u(x)\|^{2} \leq c_{5}(\log t) \operatorname{tr}\left(f^{1 / 2} x f^{1 / 2} f x^{*}\right) .
$$

A fortiori for all $x$ in $E$ we have

$$
\left\|u_{1}(x)\right\|^{2} \leq c_{5}(\log t) \operatorname{tr}\left(f^{1 / 2} x f^{1 / 2} x^{*}\right)
$$

Now (3.8), (3.5) and (3.5)' yield (3.4).

Proof of Corollary 3.5. By (3.4) we have

$$
\sum_{1}^{n}\left\|u\left(x_{i}\right)\right\|^{2} \leq K^{2}\left(c_{1} \log (t)\left\|\sum x_{i} \otimes \bar{x}_{i}\right\|_{\min }+c_{2} t^{-1}\left\|\sum x_{i} x_{i}^{*}\right\|+c_{2} t^{-1}\left\|\sum x_{i}^{*} x_{i}\right\|\right) .
$$

It is elementary that $\left\|x_{i}\right\|^{2} \leq\left\|\sum_{1}^{n} x_{i} \otimes \bar{x}_{i}\right\|$ hence $\left\|\sum x_{i} x_{i}^{*}\right\| \leq \sum\left\|x_{i}\right\|^{2} \leq n\left\|\sum x_{i} \otimes \bar{x}_{i}\right\|$, and similarly $\left\|\sum x_{i}^{*} x_{i}\right\| \leq n\left\|\sum x_{i} \otimes \bar{x}_{i}\right\|$. Therefore, choosing $t=n$, we obtain (3.3).

Corollary 3.7. Let $E_{n} \subset B(H)$ be a subspace $\lambda$-completely isomorphic to $O H_{n}$, for some $\lambda \geq 1$. Then for any projection $P: B(H) \rightarrow E_{n}$ we have

$$
\|P\|_{c b} \geq\left(c_{6}\right)^{-1} n^{1 / 2}(1+\log (n))^{-1 / 2} \lambda^{-1} .
$$

Proof. Let $v: E_{n} \rightarrow O H_{n}$ be an isomorphism such that $\|v\|_{c b}\left\|v^{-1}\right\|_{c b}=\lambda$. Let $u=$ $v P: B(H) \rightarrow O H_{n}$. Using the notation of [P6, p. 88], (3.3) implies $\pi_{2, o h}^{n}(u) \leq K\left(c_{1} \log (n)+\right.$ $1)^{1 / 2}$ hence by the estimates of $K$ in the last part of Corollary 3.4

$$
\pi_{2, o h}^{n}(u) \leq 2^{9 / 4}\left(c_{1} \log (n)+1\right)^{1 / 2}\|u\|_{c b} \leq 2^{9 / 4}\left(c_{1} \log (n)+1\right)^{1 / 2}\|v\|_{c b}\|P\|_{c b} .
$$


Let $i: E \rightarrow B(H)$ be the inclusion map. Note $P i=I_{E}$. Hence we find

$$
n^{1 / 2}=\pi_{2, o h}^{n}\left(I_{O H_{n}}\right)=\pi_{2, o h}^{n}\left(u i v^{-1}\right) \leq \pi_{2, o h}^{n}(u)\left\|v^{-1}\right\|_{c b} \leq 2^{9 / 4}\left(c_{1} \log (n)+1\right)^{1 / 2} \lambda\|P\|_{c b} .
$$

Remark. It remains an open problem (see [P6, Problem 10.2]) whether the logarithmic factor can be entirely removed from either (3.3) or (3.9).

\section{$\S 4$. Applications to Schur multipliers}

Let $B=B\left(\ell_{2}\right)$ and let $K \subset B$ denote the subalgebra of compact operators. Let $S_{1}$ denote the trace class, i.e. set of $x$ in $B$ such that $\operatorname{tr}|x|<\infty$, where we set $|x|=\left(x^{*} x\right)^{1 / 2}$, equipped with the trace class norm

$$
\|x\|_{1}=\operatorname{tr}|x|
$$

It is well known that $S_{1}^{*} \simeq B$ and $K^{*} \simeq S_{1}$ isometrically. This duality allows us to view $S_{1}$ as an operator space for which the preceding identities become completely isometric (see [ER1] or [P1]). We will study the Schur multipliers from $K$ to $S_{1}$ (or from $B$ to $S_{1}$ ), that

is to say the linear maps of the form $M_{\varphi}:\left(x_{i j}\right) \rightarrow\left(\varphi_{i j} x_{i j}\right)$ where $\left(\varphi_{i j}\right)$ is a matrix with complex entries.

The following is a rather easy consequence of $[\mathrm{P} 4]$.

Theorem 4.1. A Schur multiplier $M_{\varphi}$ is bounded from $B$ to $S_{1}$ (or from $K$ to $S_{1}$ ) iff there is a decomposition

$$
\varphi_{i j}=a_{i j}+b_{i j}
$$

with $\sum_{i} \sup _{j}\left|a_{i j}\right|+\sum_{j} \sup _{i}\left|b_{i j}\right|<\infty$.

Proof. Assume that $M_{\varphi}: K \rightarrow S_{1}$ is bounded with norm $\leq 1$. For $a, b$ in $K$, let $u(a, b)=\left\langle M_{\varphi} a, b\right\rangle$. Assuming (say) $\left\{a_{i j} \mid i, j \geq 1\right\}$ finitely supported we have

$$
u(a, b)=\sum \varphi_{i j} a_{i j} b_{i j}
$$

By [P4], $u$ satisfies (0.1) and (0.2). As mentioned after (0.2), there exists a decomposition $u=u_{1}+u_{2}+u_{3}+u_{4}$ so that there are states such that for all $a, b$ in $K$ we have

$$
\begin{array}{ll}
\left|u_{1}(a, b)\right| \leq K\left(f_{1}\left(a a^{*}\right) g_{1}\left(b^{*} b\right)\right)^{1 / 2}, & \left|u_{2}(a, b)\right| \leq K\left(f_{2}\left(a^{*} a\right) g_{2}\left(b b^{*}\right)\right)^{1 / 2} \\
\left|u_{3}(a, b)\right| \leq K\left(f_{1}\left(a a^{*}\right) g_{2}\left(b b^{*}\right)\right)^{1 / 2}, & \left|u_{4}(a, b)\right| \leq K\left(f_{2}\left(a^{*} a\right) g_{1}\left(b^{*} b\right)\right)^{1 / 2} .
\end{array}
$$


We will use an averaging argument. Let $G$ be the group of all diagonal unitary matrices on $\ell_{2}$ equipped with its normalized Haar measure $m$.

Given a bilinear form $v: K \times K \rightarrow \mathbb{C}$ we associate to it the form

$$
\widetilde{v}(a, b)=\int_{G \times G} v\left(w_{1} a w_{2}, w_{2}^{-1} b w_{1}^{-1}\right) d m\left(w_{1}\right) d m\left(w_{2}\right) .
$$

Clearly $\|\widetilde{v}\| \leq\|v\|$. Moreover, $\widetilde{v}\left(w_{1} a w_{2}, w_{1}^{-1} b w_{2}^{-1}\right)=\widetilde{v}(a, b)$ for any $w_{1}, w_{2}$ in $G$. Therefore there is a Schur multiplier $\psi$ such that, if $a, b$ are finitely supported, we have

$$
\widetilde{v}(a, b)=\sum \psi_{i j} a_{i j} b_{i j}
$$

We now apply this averaging procedure to each of $u_{1}, u_{2}, u_{3}$ and $u_{4}$. For $u_{1}$ we obtain (by Cauchy-Schwarz):

$$
\begin{aligned}
\left|\widetilde{u}_{1}(a, b)\right| & \leq \int\left|u\left(w_{1} a w_{2}, w_{1}^{-1} b w_{2}^{-1}\right)\right| d m\left(w_{1}\right) d m\left(w_{2}\right) \\
& \leq K\left(\int f_{1}\left(w_{1} a a^{*} w_{1}^{-1}\right) d m\left(w_{1}\right)\right)^{1 / 2}\left(\int g_{1}\left(w_{2} b^{*} b w_{2}^{-1}\right) d m\left(w_{2}\right)\right)^{1 / 2} .
\end{aligned}
$$

Moreover $\int w_{1}^{-1} f_{1} w_{1} d m\left(w_{1}\right)$ and $\int w_{2}^{-1} g_{1} w_{2} d m\left(w_{2}\right)$ are diagonal states on $K$. Setting $x_{i}^{1}=\left(f_{1}\right)_{i i}$ and $y_{j}^{1}=\left(g_{1}\right)_{j j}$ and $\varphi_{i j}^{1}=\widetilde{u}_{1}\left(e_{i j}, e_{i j}\right)$ we find

$$
\left|\sum \varphi_{i j}^{1} a_{i j} b_{i j}\right| \leq K\left(\sum_{i j} x_{i}^{1}\left|a_{i j}\right|^{2} \cdot \sum_{i j}\left|a_{i j}\right|^{2} y_{j}^{1}\right)^{1 / 2}
$$

Hence $\left|\varphi_{i j}^{1}\right| \leq K\left|x_{i}^{1}\right|^{1 / 2}\left|y_{j}^{1}\right|^{1 / 2}$ with

$$
\sum\left|x_{i}^{1}\right|=1, \quad \sum\left|y_{j}^{1}\right|=1
$$

Applying the same procedure successively to $u_{2}, u_{3}$ and $u_{4}$, we obtain a decomposition $\varphi=\varphi^{1}+\varphi^{2}+\varphi^{3}+\varphi^{4}$ where $\varphi^{2}$ satisfies (by symmetry) a similar bound as $\varphi^{1}$ and $\varphi^{3}, \varphi^{4}$ are such that there are $x_{i}^{3}, y_{i}^{3}, x_{i}^{4}, y_{i}^{4}$ all $\geq 0$ and with $\sum x_{i}^{3}=\sum y_{i}^{3}=\sum x_{i}^{4}=\sum y_{i}^{4}=1$ such that $\left|\varphi_{i j}^{3}\right| \leq K\left(x_{i}^{3} y_{i}^{3}\right)^{1 / 2}$ and $\left|\varphi_{i j}^{4}\right| \leq K\left(x_{j}^{4} y_{j}^{4}\right)^{1 / 2}$. Clearly we have $\sum_{i} \sup _{j}\left|\varphi_{i j}^{3}\right| \leq K$ and $\sum_{j} \sup _{i}\left|\varphi_{i j}^{4}\right| \leq K$ 
On the other hand, we claim that $\varphi^{1}$ and $\varphi^{2}$ each admit a decomposition of the kind described in Theorem 4.1. Indeed, it suffices to prove this with a uniform bound when $\varphi^{1}, x^{1}, y^{1}$ are all finitely supported. Then, after a suitable permutation of the indices, we may assume that $x_{i}^{1}$ and $y_{j}^{1}$ are both non-decreasing. Let then $\alpha_{i j}=\varphi_{i j}^{1}$ if $i \leq j$ and $\beta_{i j}=$ $\varphi_{i j}^{1}$ if $i>j$. We have then $\alpha_{i j} \leq\left(x_{i}^{1} y_{j}^{1}\right)^{1 / 2} \leq\left(x_{j}^{1} y_{j}^{1}\right)^{1 / 2}$ and $\beta_{i j} \leq\left(x_{i}^{1} y_{j}^{1}\right)^{1 / 2} \leq\left(x_{i}^{1} y_{i}^{1}\right)^{1 / 2}$ hence we obtain $\varphi_{i j}^{1}=\alpha_{i j}+\beta_{i j}$ with

$$
\sum_{j} \sup _{i}\left|\alpha_{i j}\right| \leq 1 \quad \text { and } \quad \sum_{i} \sup _{j}\left|\beta_{i j}\right| \leq 1
$$

which proves our claim. Using this we finally obtain a decomposition $\varphi_{i j}=a_{i j}+b_{i j}$ with $\sum_{i} \sup _{j}\left|a_{i j}\right| \leq K+2$ and $\sum_{j} \sup _{i}\left|b_{i j}\right| \leq K+2$. This proves the "only if" part.

Conversely, if $\varphi$ can be decomposed as in Theorem 4.1, we will show that $M_{\varphi}: B \rightarrow S_{1}$ is bounded. It clearly suffices to show that $\sum_{i} \sup _{j}\left|\varphi_{i j}\right|<\infty$ and $\sum_{j} \sup _{i}\left|\varphi_{i j}\right|<\infty$ are sufficient conditions for the boundedness of $M_{\varphi}: B \rightarrow S_{1}$. But this is obvious since:

$$
\left|\sum \varphi_{i j} a_{i j} b_{i j}\right| \leq \sum_{i} \sup _{j}\left|\varphi_{i j}\right| \sum_{j}\left|a_{i j} b_{i j}\right| \leq \sum_{i} \sup _{j}\left|\varphi_{i j}\right|\|a\|_{B}\|b\|_{B}
$$

and similarly

$$
\left|\sum \varphi_{i j} a_{i j} b_{i j}\right| \leq \sum_{j} \sup _{i}\left|\varphi_{i j}\right|\|a\|_{B}\|b\|_{B}
$$

We now turn to the c.b. analogue of the preceding statement.

Theorem 4.2. Consider a complex matrix $\left(\varphi_{i j}\right)$. The following assertions are equivalent:

(i) $M_{\varphi}$ is c.b. from $K$ to $S_{1}$.

(i) $M_{\varphi}$ is c.b. from $B$ to $S_{1}$.

(ii) There are $x, y$ in $\ell_{2}$ and a constant $C$ such that $\left|\varphi_{i j}\right| \leq C\left|x_{i}\right|\left|y_{j}\right|$ for all $i, j$.

(iii) There is a element $T$ in $S_{1}$ and a constant $C$ such that $\left|\varphi_{i j}\right| \leq C\left|T_{i j}\right|$ forall $i, j$.

Proof. The main implication is (i) $\Rightarrow$ (ii). The fact that (ii) $\Leftrightarrow$ (iii) is elementary; we include it for the record. Assume (i). Let $u: K \times K \rightarrow \mathbb{C}$ be as before with $\|u\|_{c b} \leq 1$. By 
Theorem 0.5 , there is a decomposition $u=u_{1}+u_{2}$ and states $f_{1}, f_{2}, g_{1}, g_{2}$ such that, for some constant $C$ we have for all $a, b$ in $K$ :

$$
\begin{aligned}
& \left|u_{1}(a, b)\right| \leq C\left(f_{1}\left(a a^{*}\right) g_{1}\left(b^{*} b\right)\right)^{1 / 2} \\
& \left|u_{2}(a, b)\right| \leq C\left(f_{2}\left(a^{*} a\right) g_{2}\left(b b^{*}\right)\right)^{1 / 2} .
\end{aligned}
$$

Applying the same averaging procedure as above, we find a decomposition $u=\widetilde{u}=\widetilde{u}_{1}+\widetilde{u}_{2}$ and matrices $\varphi^{1}$ and $\varphi^{2}$ such that $\widetilde{u}_{1}=M_{\varphi^{1}}$, and $\widetilde{u}_{2}=M_{\varphi^{2}}$. Moreover, we have

$$
\begin{aligned}
& \left|\sum \varphi_{i j}^{1} a_{i j} b_{i j}\right| \leq C\left(\sum_{i j} x_{i}^{1}\left|a_{i j}\right|^{2} \sum_{i j} y_{j}^{1}\left|a_{i j}\right|^{2}\right)^{1 / 2} \\
& \left|\sum \varphi_{i j}^{2} a_{i j} b_{i j}\right| \leq C\left(\sum_{i j} x_{j}^{2}\left|a_{i j}\right|^{2} \sum_{i j} y_{i}^{2}\left|a_{i j}\right|^{2}\right)^{1 / 2}
\end{aligned}
$$

where $x_{i}^{1}=\left(f_{1}\right)_{i i}, x_{j}^{2}=\left(f_{2}\right)_{j j}, y_{j}^{1}=\left(g_{1}\right)_{j j}$ and $y_{i}^{2}=\left(g_{2}\right)_{i i}$. This gives us

$$
\left|\varphi_{i j}\right|=\left|\varphi_{i j}^{1}+\varphi_{i j}^{2}\right| \leq C\left(x_{i}^{1} y_{j}^{1}+x_{j}^{2} y_{i}^{2}\right)^{1 / 2}
$$

hence if we set $x_{i}=\left(\left|x_{i}^{1}\right|+\left|y_{i}^{2}\right|\right)^{1 / 2}$ and $y_{j}=\left(\left|y_{j}^{1}\right|+\left|x_{j}^{2}\right|\right)^{1 / 2}$, we obtain

$$
\left|\varphi_{i j}\right| \leq C x_{i} y_{j}
$$

This shows that (i) $\Rightarrow$ (ii). (ii) $\Rightarrow$ (iii) is obvious. The converse is elementary. Indeed, if $T=\sum_{k} x^{k} \otimes y^{k} \lambda_{k}$ with $x^{k}, y^{k}$ in the unit ball of $\ell_{2}$ and $\sum\left|\lambda_{k}\right|<\infty$, we have

$$
\left|T_{i j}\right| \leq X_{i} Y_{j} \quad \text { where } \quad X_{i}=\left(\sum_{k} \lambda_{k}\left|x_{i}^{k}\right|^{2}\right)^{1 / 2}
$$

and $Y_{j}=\left(\sum_{k} \lambda_{k}\left|y_{j}^{k}\right|^{2}\right)^{1 / 2}$.

It remains only to show (ii) $\Rightarrow(\mathrm{i})^{\prime}$ ( $\operatorname{since}(\mathrm{i})^{\prime} \Rightarrow$ (i) is trivial by restriction). Assume (ii). Let $f=\sum\left|x_{i}\right|^{2} e_{i i}$ and $g=\sum\left|y_{j}\right|^{2} e_{j j}$. For any finitely supported $a, b$ we have by Cauchy-Schwarz

$$
\begin{aligned}
\left|\sum \varphi_{i j} a_{i j} b_{i j}\right| & \leq C \sum\left|x_{i}\right|\left|a_{i j}\right|\left|y_{j}\right| \leq C\left(\sum_{i j}\left|x_{i}\right|^{2}\left|a_{i j}\right|^{2} \sum_{i j}\left|a_{i j}\right|^{2}\left|y_{j}\right|^{2}\right)^{1 / 2} \\
& \leq C\left(f\left(a a^{*}\right) g\left(b^{*} b\right)\right)^{1 / 2} .
\end{aligned}
$$


Hence we conclude that the bilinear from $u$ is c.b.; a fortiori $u$ is j.c.b. and $M_{\varphi}$ is c.b.

Remark. The preceding argument actually shows that the bilinear form $(a, b) \rightarrow\left\langle M_{\varphi} a, b\right\rangle$ is j.c.b. iff it is c.b.

Remark. It is easy to see that (ii) in Theorem 4.2 is equivalent to:

$(\text { ii })^{\prime}$ There are $\left(a_{i j}\right)$ and $\left(b_{i j}\right)$ with $\sum_{i} \sup _{j}\left|a_{i j}\right|+\sum_{j} \sup _{i}\left|b_{i j}\right|<\infty$ such that

$$
\left|\varphi_{i j}\right|=\sqrt{a_{i j} b_{i j}}
$$

Thus, whereas we had the arithmetic mean in Theorem 4.1, here we find the geometric mean of the same terms.

In particular, it is easy to see from this that there are bounded Schur multipliers $M_{\varphi}: K \rightarrow$ $S_{1}$ which are not c.b.

\section{References}

[B1] D. Blecher. Generalizing Grothendieck's program. "Function spaces", Edited by K. Jarosz, Lecture Notes in Pure and Applied Math. vol.136, Marcel Dekker, 1992.

[BL] J. Bergh and J. Löfström. Interpolation spaces. An introduction. Springer Verlag, New York. 1976.

[BP] D. Blecher and V. Paulsen. Tensor products of operator spaces. J. Funct. Anal. 99 (1991) 262-292.

[C] A. Connes, Almost periodic states and factors of type $\mathrm{III}_{1}$, J. Funct. Anal. 16 (1974), $415-455$.

[CES] E. Christensen, E. Effros and A. Sinclair. Completely bounded multilinear maps and $C^{*}$-algebraic cohomology. Invent. Math. 90 (1987) 279-296.

[CS1] E. Christensen and A. Sinclair. Representations of completely bounded multilinear operators. J. Funct. Anal. 72 (1987) 151-181.

[CS2] _ A survey of completely bounded operators. Bull. London Math. Soc. 21 (1989) 417-448.

[D] K. Dykema, Free products of finite dimensional and other von Neumann algebras with respect to non-tracial states, Free Probability (D.-V. Voiculescu, ed.), Fields Institute Communications, vol. 12, American Mathematical Society, 1997, pp. 41-88. 
[ER1] E. Effros and Z.J. Ruan. Operator Spaces. Oxford Univ. Press, Oxford, 2000.

[ER2] . A new approach to operator spaces. Canadian Math. Bull. 34 (1991) 329-337.

[ER3] - Self duality for the Haagerup tensor product and Hilbert space factorization. J. Funct. Anal. 100 (1991) 257-284.

[G] A. Grothendieck. Résumé de la théorie métrique des produits tensoriels topologiques. Boll.. Soc. Mat. São-Paulo 8 (1956), 1-79.

[H1] U. Haagerup. The Grothendieck inequality for bilinear forms on $C^{*}$-algebras. Adv. in Math. 56 (1985) 93-116. - Self-polar forms, conditional expectations and the weak expectation property for $C^{*}$-algebras. Unpublished manuscript (1995).

[HI] U. Haagerup and T. Itoh. Grothendieck type norms for bilinear forms on $C^{*}$-algebras. J. Operator Theory 34 (1995) 263-283.

[J] M. Junge. A first attempt to the little Grothendieck inequality and related embeddings. Unpublished notes from a lecture at I.H.P. (January 2000).

[JP] M. Junge and G. Pisier. Bilinear forms on exact operator spaces and $B(H) \otimes B(H)$. Geometric and Functional Analysis (GAFA Journal) 5 (1995) 329-363.

[Ki1] E. Kirchberg. On non-semisplit extensions, tensor products and exactness of group $C^{*}$-algebras. Invent. Math. 112 (1993) 449-489.

[Ki2] . Commutants of unitaries in UHF algebras and functorial properties of exactness. J. Reine Angew. Math. 452 (1994), 39-77.

$[\mathrm{Ki} 3]$ . Exact $C^{*}$-algebras, Tensor products, and Classification of purely infinite algebras. Proceedings ICM 94, Zürich, vol. 2, 943-954, Birkhauser, 1995.

[L] E.C. Lance. On nuclear $C^{*}$-algebras. J. Functional Analysis 12 (1973), 157-176.

[O] T. Oikhberg. Direct sums of homogeneous operator spaces. J. London Math. Soc. To appear.

[OP] T. Oikhberg and G. Pisier. The "maximal" tensor product of two operator spaces. Proc. Edinburgh Math. Soc. 42 (1999) 267-284.

[PaS] V. Paulsen and R. Smith. Multilinear maps and tensor norms on operator systems. 
J. Funct. Anal. 73 (1987) 258-276.

[P1] G. Pisier. Introduction to operator space theory. Cambridge Univ. Press. Book to appear.

$[\mathrm{P} 2]$ - A simple proof of a theorem of Kirchberg and related results on $C^{*}$-norms. J. Op. Theory. 35 (1996) 317-335.

[P3] . Exact operator spaces. Colloque sur les algèbres d'opérateurs. in "Recent advances in operator algebras" (Orléans 1992) Astérisque (Soc. Math. France) 232 (1995) 159-186.

$[\mathrm{P} 4]$ . Grothendieck's theorem for non-commutative $\mathrm{C}^{*}$-algebras with an appendix on Grothendieck's constants. J. Funct. Anal. 29 (1978) 397-415. $[\mathrm{P} 5]$ . Factorization of linear operators and the Geometry of Banach spaces. CBMS (Regional conferences of the A.M.S.) 60, (1986), Reprinted with corrections 1987.

[P6] . Non-commutative vector valued $L_{p}$-spaces and completely p-summing maps. Soc. Math. France. Astérisque 247 (1998) 1-131.

$[\mathrm{P} 7]$ . The operator Hilbert space $O H$, complex interpolation and tensor norms. Memoirs Amer. Math. Soc. vol. 122 , 585 (1996) 1-103.

[Sc] R. Schneider. Zonoids whose polars are zonoids. Proc. Amer. Math. Soc. 50 (1975) 365-368.

[S1] D. Shlyakhtenko. Free quasi-free states. Pacific J. Math. 177 (1997), no. 2, 329-368. $[\mathrm{S} 2]$ . Limit distributions of matrices with bosonic and fermionic entries. Free probability theory (Waterloo, ON, 1995), 241-252, Fields Inst. Commun., 12, Amer. Math. Soc., Providence, RI, 1997.

$[\mathrm{S} 3]$ . A-valued semicircular systems. J. Funct. Anal. 166 (1999), $1-47$.

[VDN] D. Voiculescu, K. Dykema, A. Nica. Free random variables. CRM Monograph Series, Vol. 1, Amer. Math. Soc., Providence RI. 